\title{
On a Bernoulli Property for Multi-dimensional Mappings with Finite Range Structure
}

\author{
Michiko YURI \\ Tsuda College
}

\section{Introduction}

In the previous paper [4], we considered ergodic properties of a mapping $T$ defined on a bounded domain $X \subset R^{d}$ satisfying a "local Renyi's condition". The purpose of this paper is to prove that such a mapping $T$ is weak Bernoulli if it admits a finite absolutely continuous invariant measure.

The mapping we consider is characterized by a certain type of partition $Q=\left\{X_{a}: a \in I\right\}$ of $X$ and a finite number of subsets $U_{0}(=X), U_{1}, \cdots, U_{N}$ of $X$ satisfying some special properties (see $\S 1$ for precise definitions). We shall call such a transformation $T$ a multi-dimensional mapping with a finite range structure. If such a $T$ satisfies the Renyi's condition, in addition, then it is known that $T$ has a finite absolutely continuous invariant measure, and furthermore, under some additional conditions one can prove that $Q$ is a weak Bernoulli partition ([9], [18]). On the other hand, when $X$ is an interval of $R^{1}$, Ledrappier established in [6] the weak Bernoulli property for a transformation $T$ having a similar characterization under some further hypothesis, such as the existence of a finite invariant measure with positive entropy, but without assuming that $T$ satisfies the Renyi's condition (cf. [2]). The main ingredient of his proof, which is patterned after the work of Sinai [15] (cf. [16]) and Ratner [12], is the use of Rohlin's formula for proving the absolute continuity of some conditional measures.

In this paper we establish a sufficient condition for a multi-dimensional mapping with a finite range structure to have the weak Bernoulli property when they do not necessarily satisfy the Renyi's condition. We do need, however, to make several assumptions on the transformation; some of these assumptions seem to be essential, while the others are seen 
to be purely technical (see $\S 1$ ). Under the assumptions explained in detail in $\S 1$, we will show that $T$ is exact $(\S 2)$, and that "Rohlin's formula" holds for $T(\S 3)$. In $\S 4$, we construct a natural extension for $T$ and consider conditional measures with respect to the extension. By using Rohlin's formula, we prove the absolute continuity of the conditional measures, and this will lead us to the desired conclusion along the line of argument used in [6] and [12]. Since the reasoning follows more or less the same pattern we will only sketch the outline of the argument used for this part.

The remainder (\$5) of the paper is devoted to the discussion of three examples of multi-dimensional mapping with a finite range structure. One of these examples is a one parameter family of maps of an interval, and the others arise from the number theory: an inhomogeneous diophantine approximation problem and complex continued fractions. None of these transformations satisfy the Renyi's condition, but all of them do satisfy a "local Renyi's condition". We will show that these transformations satisfy all of the assumptions made in $\S 1$, and therefore weak Bernoulli.

\section{§1. Notations and results.}

Definition. A mapping $T$ on a bounded domain $X \subset R^{d}$ is called " $a$ multi-dimensional mapping with a finite range structure" if there exist a countable partition $Q=\left\{X_{a}: a \in I\right\}$ of $X$ and a finite number of subsets $\left\{U_{0}, U_{1}, \cdots, U_{N}\right\}$ of $X$ satisfying the following Conditions (1) (4):

(1) Each $X_{a}$ is a measurable connected subset with piecewise smooth boundary.

(2) Each $U_{k}$ has a positive measure.

(3) For each $X_{a}$ the mapping $T_{X_{a}}^{1_{a}}$ restricted on $X_{a}$ is injective, of class $C^{1}$, and $\operatorname{det} D T_{X_{a}}^{1} \neq 0$.

(4) If $\operatorname{int}\left(X_{a_{1}}\right) \cap \operatorname{int}\left(T^{-1} X_{a_{2}}\right) \cap \cdots \cap \operatorname{int}\left(T^{-(n-1)} X_{a_{n}}\right) \neq \varnothing$, then $T^{n}\left(X_{a_{1}} \cap\right.$ $\left.T^{-1} X_{a_{2}} \cap \cdots \cap T^{-(n-1)} X_{a_{n}}\right)=U_{k}$ for some $k \in\{0,1, \cdots, N\}$.

To state our results, we introduce some notations. If $\operatorname{int}\left(X_{a_{1}}\right) \cap$ $\operatorname{int}\left(T^{-1} X_{a_{2}}\right) \cap \cdots \cap \operatorname{int}\left(T^{-(n-1)} X_{a_{n}}\right) \neq \varnothing$, we denote $X_{a_{1}} \cap T^{-1} X_{a_{2}} \cap \cdots \cap T^{-(n-1)} X_{a_{n}}$, by $X_{a_{1} \cdots a_{n}}$, and call it a cylinder of rank $n$ with respect to $T$. $\mathscr{L}^{(n)}$ denotes the family of all cylinders $X_{a_{1} \cdots a_{n}}$ of rank $n$, and $\mathscr{L}=\cup_{n=1}^{\infty} \mathscr{L}^{(n)}$. If $X_{a_{1} \cdots a_{n}} \in \mathscr{L}^{(n)}$, we call the sequence $\left(a_{1} \cdots a_{n}\right) T$-admissible. Denote the set of all $T$-admissible sequences of length $n$ by $A(n)$. We write $\Psi_{a}$ for $\left(T_{X_{a}}^{\prime}\right)^{-1}$ and define inductively

$$
\Psi_{a_{1} \cdots a_{n}}=\Psi_{a_{1} \cdots a_{n-1}} \circ \Psi_{a_{n}} \text {. }
$$


For a constant $C \geqq 1$, we call a cylinder $X_{a_{1} \cdots a_{n}}$ an "R.C-cylinder" if it satisfies "Renyi's condition", i.e.

$$
\sup _{x \in T^{n} X_{a_{1} \cdots a_{n}}}\left|\operatorname{det} D \Psi_{a_{1} \cdots a_{n}}(x)\right| \leqq C \cdot \inf _{x \in T^{n} X_{a_{1} \cdots a_{n}}}\left|\operatorname{det} D \Psi_{a_{1} \cdots a_{n}}(x)\right| \text {. }
$$

Let $R(C . T)$ denote the set of all $R . C$-cylinders. We define for $C \geqq 1$

$$
\begin{aligned}
& \mathscr{D}_{n}=\left\{X_{a_{1} \cdots a_{n}} \in \mathscr{L}^{(n)}: X_{a_{1} \cdots a_{j}} \in \mathscr{L} \backslash R(C . T) \text { for } 1 \leqq j \leqq n\right\}, \\
& D_{n}=\bigcup_{X_{a_{1} \cdots a_{n} \in \mathscr{D}_{n}}} X_{a_{1} \cdots a_{n}}, \\
& \beta_{n}=\left\{X_{a_{1} \cdots a_{n}} \in \mathscr{L}^{(n)}: X_{a_{1} \cdots a_{n-1}} \in \mathscr{D}_{n-1}, X_{a_{1} \cdots a_{n}} \in R(C . T)\right\}, \\
& B_{n}=\bigcup_{X_{a_{1} \cdots a_{n} \in \beta_{n}}} X_{a_{1} \cdots a_{n}} .
\end{aligned}
$$

Let $\lambda(\cdot)$ be the normalized Lebesgue measure on $X$.

We now state some conditions to be used in our results.

(C.1) (generator condition)

$\vee_{m=1}^{\infty} T^{-m} Q=\varepsilon$, i.e. the partition into points.

Assume that there exists a constant $C \geqq 1$ such that

(C.2) (transitivity condition)

for each $j$ with $0 \leqq j \leqq N$, there exists a cylinder $X_{a_{1} \cdots a_{s j}}$ contained

in $U_{j}$ such that $X_{a_{1} \cdots a_{s j}} \in R(C . T)$ and $T^{s_{j}} X_{a_{1} \cdots a_{s j}}=X$,

(C.3) if $X_{a_{1} \cdots a_{n}} \in R(C . T)$, then $X_{b_{1} \cdots b_{k} a_{1} \cdots a_{n}} \in R(C . T)$ for any

$\left(b_{1} \cdots b_{k} a_{1} \cdots a_{n}\right) \in A(k+n)$,

(C.4) $\sum_{n=1}^{\infty} \lambda\left(D_{n}\right)<+\infty$.

Under the above conditions, we have

ThEOREM 1. T is exact.

REMARK 1. In previous paper [4], we showed that $T$ is ergodic and has a finite invariant measure $\mu$ equivalent to $\lambda$ under the same conditions (C.1) (C.4).

Assume further

(C.5) for all $n>0$,

$$
\begin{aligned}
W_{n} & \equiv \sum_{m=0}^{\infty}\left(\sum _ { X _ { k _ { 1 } \cdots k _ { m } } \in \mathscr { D } _ { m } } \left(\sup _{\left.y \in T^{m} X_{k_{1} \cdots k_{m}} \cap\left({\underset{j=1}{\cup} B_{j} j}_{j}\left|\operatorname{det} D \Psi_{k_{1} \cdots k_{m}}(y)\right|\right)\right)}\right.\right. \\
& <+\infty,
\end{aligned}
$$

(C.6) $\mathscr{D}_{1}<+\infty$,

(C.7) there exists a positive integer $l$ such that for all $n>0$ and all $X_{a_{1} \cdots a_{n}} \in \mathscr{D}_{n}$ 


$$
\frac{\sup _{x \in T^{n} X_{a_{1} \cdots a_{n}}}\left|\operatorname{det} D \Psi_{a_{1} \cdots a_{n}}(x)\right|}{\inf _{x \in T n^{n} X_{a_{1} \cdots a_{n}}}\left|\operatorname{det} D \Psi_{a_{1} \cdots a_{n}}(x)\right|}=O\left(n^{l}\right),
$$

(C.8) $\log |\operatorname{det}(D T(\cdot))| \in \mathscr{L}^{1}(X, \lambda)$.

Then we have

THEOREM 2. Rohlin's formula (R) is true.

$$
\text { (R): } \quad h(T)=\int_{X} \log |\operatorname{det} D T(x)| d \mu(x) .
$$

REMARK 2. In general, the density of $\mu$ is not bounded (for example, see §5). For this reason, we need some technical Conditions (C.5) and (C.6). These conditions allow us to have the following properties: The density of $\mu$ is bounded on $\left(D_{n}\right)^{\circ}$ for each $n>0$, and therefore for all $n>0$, there exists $M(n)$ such that

$$
\frac{\mu\left(X_{a_{1} \cdots a_{n}}\right)}{\lambda\left(X_{a_{1} \cdots a_{n}}\right)}<M(n) \text { for any } X_{a_{1} \cdots a_{n}} \in \mathscr{L}^{(n)} .
$$

(This is proved in $\S 3$ ).

REMARK 3. (C.7) is a weaker Renyi's condition. It is easy to see that we can replace $\mathscr{D}_{n}$ by $\mathscr{L}^{(n)}$, and this condition allows us to have

$$
\inf _{x \in X}|\operatorname{det} D T(x)|>0 \text {. }
$$

For main theorem, we also suppose

(C.4)* $\sum_{n=1}^{\infty} \lambda\left(D_{n}\right) \log n<+\infty$,

(C.9) there exists a positive integer $k_{0}$ which satisfies the following; if $X_{a_{1} \cdots a_{n}} \in \mathscr{D}_{n}^{c}$ and $X_{a_{2} \cdots a_{n}} \in \mathscr{D}_{n-1}$, then

$$
X_{a_{1} \cdots a_{n}} \subset \bigcup_{j=1}^{k_{0}} B_{j} .
$$

Theorem 3. Let $T$ be a multi-dimensional mapping with a finite range structure satisfying (C.1) (C.9). Then $Q$ is a weak Bernoulli partition with respect to $T$.

\section{§2. Proof of Theorem 1 .}

From a basic fact proved in Rohlin's paper [13], it is sufficient to show that for all measurable sets $E$ of positive measure with measurable images $T E, T^{2} E, \cdots$, 


$$
\lim _{n \rightarrow \infty} \mu\left(T^{n} E\right)=\mu(X)
$$

Let $\varepsilon$ be a positive number. By Lemma 2.1 in $\S 2$ of [4], the reexists $X_{a_{1} \cdots a_{n}} \in R(C . T)$ such that

$$
\lambda\left(E \cap X_{a_{1} \cdots a_{n}}\right)>(1-\varepsilon) \lambda\left(X_{a_{1} \cdots a_{n}}\right),
$$

and therefore

$$
\varepsilon \cdot \lambda\left(X_{a_{1} \cdots a_{n}}\right)>\lambda\left(X_{a_{1} \cdots a_{n}} \cap E^{c}\right)
$$

It follows from (2.1) and relation

$$
\lambda\left(T^{n} X_{a_{1} \cdots a_{n}} \cap\left(T^{n} E\right)^{\bullet}\right) \leqq \int_{E^{\mathscr{c}} \cap X_{a_{1} \cdots a_{n}}}\left|\operatorname{det} D T^{n}(x)\right| d \lambda(x)
$$

that

$$
\lambda\left(T^{n} X_{a_{1} \cdots a_{n}} \cap\left(T^{n} E\right)^{c}\right)<C \cdot \varepsilon \cdot \lambda\left(T^{n} X_{a_{1} \cdots a_{n}}\right) .
$$

From (C.2), for $T^{n} X_{a_{1} \cdots a_{n}}=U_{j}$, there exists $X_{a_{1} \cdots a_{s j}} \subset U_{j}$ such that

$$
X_{a_{1} \cdots a_{s j}} \in R(C . T) \text { and } T^{s_{j}} X_{a_{1} \cdots a_{s j}}=X \text {, }
$$

hence

$$
\lambda\left(X_{a_{1} \cdots a_{s j}} \cap\left(T^{n} E\right)^{c}\right)<C \cdot \varepsilon \cdot \lambda\left(T^{n} X_{a_{1} \cdots a_{n}}\right) .
$$

Put $D=\min _{0 \leqq j \leqq N}\left(\lambda\left(X_{a_{1} \cdots a_{s j}}\right) / \lambda\left(U_{j}\right)\right)$, then

$$
\lambda\left(X_{a_{1} \cdots a_{s j}} \cap\left(T^{n} E\right)^{c}\right)<\frac{C \cdot \varepsilon}{D} \lambda\left(X_{a_{1} \cdots a_{s j}}\right) .
$$

By virtue of the properties of $X_{a_{1} \cdots a_{s j}}$, it follows from (2.4) that

$$
\begin{aligned}
\lambda\left(T^{s_{j}}\right. & \left.\left(X_{a_{1} \cdots a_{s_{j}}} \cap\left(T^{n} E\right)^{\circ}\right)\right) \\
& \leqq C\left\{\inf _{x \in X_{a_{1} \cdots a_{s_{j}}}}\left|\operatorname{det} D T^{s_{j}}(x)\right|\right\} \lambda\left(X_{a_{1} \cdots a_{s j}} \cap\left(T^{n} E\right)^{c}\right) \\
& <\frac{C^{2} \cdot \varepsilon}{D} \cdot \lambda\left(X_{a_{1} \cdots a_{s j}}\right) \cdot \inf _{x \in X_{a_{1} \cdots a_{s j}}}\left|\operatorname{det} D T^{s_{j}}(x)\right| \\
& <\frac{C^{2} \cdot \varepsilon}{D} .
\end{aligned}
$$

Using the equality

$$
\lambda\left(T^{s_{j}}\left(X_{a_{1} \cdots a_{s_{j}}} \cap\left(T^{n} E\right)^{c}\right)\right)=1-\lambda\left(T^{s_{j}}\left(X_{a_{1} \cdots a_{s_{j}}} \cap T^{n} E\right)\right),
$$

we obtain 
462

$$
\lambda\left(T^{n+\bullet_{j}} E\right)>\lambda\left(T^{\varepsilon_{j}}\left(X_{a_{1} \cdots a_{\varepsilon j}} \cap T^{n} E\right)\right)>1-\frac{C^{2} \cdot \varepsilon}{D} .
$$

From this and the fact that $\mu \sim \lambda$, the theorem follows (cf. [4]).

§3. The proof of Theorem 2.

We now prepare some lemmas to be used in the proof of Theorem 2. We note that in [4] $T$-invariant ergodic measure $\mu$ such that $\mu \sim \lambda$ was given by

$$
\mu(A)=\sum_{m=0}^{\infty} \nu\left(T^{-m} A \cap D_{m}\right)
$$

for any measurable set $A$, where $\nu \sim \lambda$ and there is a constant $G>1$ satisfying

$$
G^{-1} \leqq \frac{d \nu}{d \lambda} \leqq G
$$

As we have announced in introduction, we first prove

LEMMA 3.1. There exists a monotone increasing sequence $\{\hat{M}(n)\}_{n>0}$ such that for any $X_{a_{1} \cdots a_{n+k}} \subset B_{n}$

$$
\frac{\mu\left(X_{a_{1} \cdots a_{n+k}}\right)}{\lambda\left(X_{a_{1} \cdots a_{n+k}}\right)}<\hat{M}(n)
$$

Furthermore, we have for all $n>0$ and $X_{a_{1} \cdots a_{n}} \in \mathscr{L}^{(n)}$,

$$
G^{-1} \leqq \frac{\mu\left(X_{a_{1} \cdots a_{n}}\right)}{\lambda\left(X_{a_{1} \cdots a_{n}}\right)} \leqq M(n),
$$

where

$$
M(n)=\max \left\{\hat{M}(n), \max _{X_{a_{1} \cdots a_{n} \in \mathscr{S}_{n}}}\left\{\frac{\mu\left(X_{a_{1} \cdots a_{n}}\right)}{\lambda\left(X_{a_{1} \cdots a_{n}}\right)}\right\}\right\} .
$$

Proof. We note that the following equality is true.

$$
X_{k_{1} \cdots k_{m} a_{1} \cdots a_{n}}=\Psi_{k_{1} \cdots k_{m}}\left(T^{m} X_{k_{1} \cdots k_{m}} \cap X_{a_{1} \cdots a_{n}}\right) .
$$

From this, we have

$$
\begin{aligned}
\mu\left(X_{a_{1} \cdots a_{m+k}}\right) & \leqq G \sum_{m=0}^{\infty}\left(\sum_{x_{k_{1} \cdots \cdot k_{m}} \in \mathscr{S}_{m}} \lambda\left(X_{k_{1} \cdots k_{m} a_{1} \cdots a_{n+k}}\right)\right) \\
& =G \sum_{m=0}^{\infty}\left(\sum_{X_{k_{1} \cdots k_{m}} \in \mathscr{S}_{m}}\left(\int_{T^{m_{X}} X_{k_{1} \cdots k_{m}} \cap X_{a_{1} \cdots a_{n}+k}} \operatorname{det} D \Psi_{k_{1} \cdots k_{m}}(x) \mid d \lambda(x)\right)\right)
\end{aligned}
$$


$\leqq G \sum_{m=0}^{\infty}\left(\sum_{X_{k_{1}} \cdots k_{m} \in \mathscr{T}_{m}}\left(\sup _{x \in T^{m} X_{k_{1} \cdots k_{m}} \cap X_{a_{1} \cdots a_{n+k}}}\left|\operatorname{det} D \Psi_{k_{1} \cdots k_{m}}(x)\right| \cdot \lambda\left(T^{m} X_{k_{1} \cdots k_{m}} \cap X_{a_{1} \cdots a_{n+k}}\right)\right)\right)$ $\leqq G \sum_{m=0}^{\infty}\left(\sum_{X_{k_{1} \cdots k_{m} \in \mathscr{T}_{m}}}\left(\sup _{x \in T^{m} X_{K_{1} \cdots k_{m}} \cap X_{a^{1} \cdots o_{n+k}}}\left|\operatorname{det} D \Psi_{k_{1} \cdots k_{m}}(x)\right| \cdot \lambda\left(X_{a_{1} \cdots a_{n+k}}\right)\right)\right.$.

If $X_{a_{1} \cdots a_{n+k}} \subset B_{n}$, then

$$
\mu\left(X_{a_{1} \cdots a_{n+k}}\right) \leqq G \cdot W_{n} \cdot \lambda\left(X_{a_{1} \cdots a_{n+k}}\right) .
$$

Taking $G \cdot W_{n}$ for $\hat{M}(n)$, we obtain the first statement. Note that $X_{a_{1} \cdots a_{n}} \in \mathscr{D}_{n}^{e}$ implies $X_{a_{1} \cdots a_{n}} \subset \cup_{j=1}^{n} B_{j}$. Then, from (C.6) the second assertion is verified immediately.

The Conditions (C.7) and (C.8) allow us to have the following properties:

LEMMA 3.2. (3.2-a) $\log |\operatorname{det} D T(x)| \in \mathscr{L}^{1}(X, \mu)$,

(3.2-b) for all $n>0$ and $j \in\{0, \cdots, N\}$, put

$$
c^{j}(n) \equiv-\sum_{\substack{\left(a_{1} \cdots a_{n}\right) ; \\ X_{a_{1} \cdots a_{n}} \cap U_{j} \neq \varnothing}} \lambda\left(X_{a_{1} \cdots a_{n}}\right) \log \lambda\left(X_{a_{1} \cdots a_{n}} \cap U_{j}\right) .
$$

Then $c^{j}(n)<+\infty$.

(3.2-c) $H(Q)=-\sum_{a} \mu\left(X_{a}\right) \log \mu\left(X_{a}\right)<+\infty$.

Remark 4. Since (C.2) implies $X \in\left\{U_{0}, \cdots, U_{N}\right\}$, (3.2-b) allows us to have for all $n>0-\sum \lambda\left(X_{a_{1} \cdots a_{n}}\right) \log \lambda\left(X_{a_{1} \cdots a_{n}}\right)<+\infty$.

Proof. Here we denote $T_{X_{a}}$ by $T_{a}$. Since

$$
\int_{X} \max (0, \log |\operatorname{det} D T(x)|) d \mu(x)=\sum_{a} \int_{X_{a}} \max \left(0, \log \left|\operatorname{det} D T_{a}(x)\right|\right) d \mu(x),
$$

and

$$
\sup _{x \in X_{a}}\left(\log \left|\operatorname{det} D T_{a}(x)\right|\right) \leqq \log \left(\sup _{x \in X_{a}}\left|\operatorname{det} D T_{a}(x)\right|\right),
$$

it follows from (C.7) and (3.1) that

$$
\begin{aligned}
\int_{X} & \max (0, \log |\operatorname{det} D T(x)|) d \mu(x) \leqq \sum_{a} \max \left(0, \mu\left(X_{a}\right) \log \left(\sup _{x \in X_{a}}\left|\operatorname{det} D T_{a}(x)\right|\right)\right) \\
& \leqq \sum_{a} \max \left(0, \mu\left(X_{a}\right) \log \left(C_{1} \inf _{x \in X_{a}}\left|\operatorname{det} D T_{a}(x)\right|\right)\right) \\
& \leqq \sum_{a} \max \left(0, M(1) \cdot \lambda\left(X_{a}\right) \log C_{1}+M(1) \lambda\left(X_{a}\right) \log \left(\inf _{x \in X_{a}}\left|\operatorname{det} D T_{a}(x)\right|\right)\right) \\
& \leqq \sum_{a} M(1) \lambda\left(X_{a}\right) \log C_{1}+\sum_{a} \max \left(0, M(1) \lambda\left(X_{a}\right) \log \left(\inf _{x \in X_{a}}\left|\operatorname{det} D T_{a}(x)\right|\right)\right),
\end{aligned}
$$


where $C_{1}$ is a constant such that

$$
\frac{\sup _{x \in T X_{a}}\left|\operatorname{det} D \Psi_{a}(x)\right|}{\inf _{x \in T X_{a}}\left|\operatorname{det} D \Psi_{a}(x)\right|}<C_{1}
$$

for all $X_{a} \in \mathscr{L}^{(1)}$.

On the other hand, using the inequality

$$
\log \left(\inf _{x \in X_{a}}\left|\operatorname{det} D T_{a}(x)\right|\right) \leqq \inf _{x \in X_{a}}\left(\log \left|\operatorname{det} D T_{a}(x)\right|\right),
$$

we have

$$
\sum_{a} \max \left(0, \log \left(\inf _{x \in X_{a}}\left|\operatorname{det} D T_{a}(x)\right|\right) \cdot \lambda\left(X_{a}\right)\right) \leqq \int_{x} \max (0, \log |\operatorname{det} D T(x)|) d \lambda .
$$

From this and (C.8), we obtain

$$
\begin{aligned}
\int_{X} \max (0, \log |\operatorname{det} D T(x)|) d \mu(x) \\
\quad<M(1) \log C_{1}\left(\sum_{a} \lambda\left(X_{a}\right)\right)+M(1) \int_{X} \max (0, \log |\operatorname{det} D T(x)|) d \lambda \\
\quad<+\infty .
\end{aligned}
$$

Now we remark that $E \equiv \inf _{x \in X}|\operatorname{det} D T(x)|>0$. In fact, the relation

$$
\lambda\left(T_{a} X_{a}\right)=\int_{X_{a}}\left|\operatorname{det} D T_{a}(x)\right| d \lambda \leqq C_{1} \cdot \inf _{x \in X_{a}}\left|\operatorname{det} D T_{a}(x)\right| \cdot 1
$$

allows us to have

$$
\inf _{x \in X_{a}}\left|\operatorname{det} D T_{a}(x)\right| \geqq \frac{L}{C_{1}} \quad \text { for all } X_{a} \in \mathscr{L}^{(1)},
$$

where $L \equiv \min _{0 \leq j \leq N} \lambda\left(U_{j}\right)$. This implies $\inf _{x \in X}|\operatorname{det} D T(x)|>0$. From this, we have immediately

$$
\int_{X} \min (0, \log |\operatorname{det} D T(x)|) d \mu(x) \geqq \int_{X} \min (0, \log E) d \mu(x)>-\infty .
$$

Therefore, combining the above results, we conclude (3.2-a).

By the condition (C.7), we have for all $n>0$ a constant $C_{n}$ such that

$$
\sup _{x \in T^{n} X_{a_{1} \cdots a_{n}}}\left|\operatorname{det} D \Psi_{a_{1} \cdots a_{n}}(x)\right|<C_{n} \cdot \inf _{x \in T^{n} X_{a_{1} \cdots a_{n}}}\left|\operatorname{det} D \Psi_{a_{1} \cdots a_{n}}(x)\right|
$$

for any $X_{a_{1} \cdots a_{n}} \in \mathscr{L}^{(n)}$. Using this and the equality 


$$
\int_{X_{a_{1} \cdots a_{n}}} \log \left|\operatorname{det} D T^{n}(x)\right| d \lambda=\int_{X_{a_{1} \cdots a_{n}}} \log \left(\frac{1}{\left|\operatorname{det} D \Psi_{a_{1} \cdots a_{n}}\left(T^{n} x\right)\right|}\right) d \lambda,
$$

we obtain

$$
\begin{aligned}
\int_{X} \log \left|\operatorname{det} D T^{n}(x)\right| d \lambda(x) \\
\quad \geqq \sum_{\left(a_{1} \cdots a_{n}\right) \in A(n)} \lambda\left(X_{a_{1} \cdots a_{n}}\right) \log \left(\frac{1}{\operatorname{supp}_{x \in T^{n} X_{a_{1} \cdots a_{n}}}\left|\operatorname{det} D \Psi_{a_{1} \cdots a_{n}}(x)\right|}\right) \\
\quad \geqq \sum_{\left(a_{1} \cdots a_{n}\right) \in A(n)} \lambda\left(X_{a_{1} \cdots a_{n}}\right) \log \left(\frac{1}{C_{n}{ }_{x \in T^{n} X_{a_{1} \cdots a_{n}}} \inf _{a_{1}}\left|\operatorname{det} D \Psi_{a_{1} \cdots a_{n}}(x)\right|}\right) .
\end{aligned}
$$

Note that for all $U_{j}$, there exists $X_{b_{1} \ldots b_{l}}{ }_{(j)}$ such that

$$
T^{l} X_{b_{1}} \ldots{ }^{(j)}=U_{j} .
$$

Therefore, if $X_{a_{1} \cdots a_{n}} \cap U_{j} \neq \varnothing$ then we have the following;

$$
\begin{aligned}
& \lambda\left(X_{a_{1} \cdots a_{n}} \cap U_{j}\right)=\int_{X_{a_{1} \cdots a_{n} \cap T^{l} X_{b_{1}} \cdots b_{l}}} d \lambda(x) \\
& =\int_{T^{n}\left(X_{a l} \cdots a_{n} \cap T^{l} X_{b_{1}} \ldots b_{l}\right)}\left|\operatorname{det} D \Psi_{a_{1} \cdots a_{n}}(x)\right| d \lambda(x) \\
& =\int_{T^{n+l}\left(X_{\left.b_{1} \cdots b_{l} a_{1} \cdots a_{n}\right)}\right.}\left|\operatorname{det} D \Psi_{a_{1} \cdots a_{n}}(x)\right| d \lambda(x) \\
& \geqq_{x \in T^{n+l}\left(X_{\left.b_{1} \cdots b_{l} a_{1} \cdots a_{n}\right)}\right.}\left|\operatorname{det} D \Psi_{a_{1} \cdots a_{n}}(x)\right| \cdot \lambda\left(T^{n+l} X_{b_{1} \cdots b_{l} a_{1} \cdots a_{n}}\right) \\
& \geqq \inf _{x \in T^{n}\left(X_{\left.a_{1} \cdots a_{n}\right)}\right)}\left|\operatorname{det} D \Psi_{a_{1} \cdots a_{n}}(x)\right| \cdot L \text {. }
\end{aligned}
$$

In particular, for $U_{j}=X$, we have $\lambda\left(X_{a_{1} \cdots a_{n}}\right) \geqq \inf \left|\operatorname{det} D \Psi_{a_{1} \cdots a_{n}}(x)\right| L$ similarly. Using the above inequality, we obtain

$$
\begin{aligned}
& \int_{X} \log \left|\operatorname{det} D T^{n}(x)\right| d \lambda(x)
\end{aligned}
$$

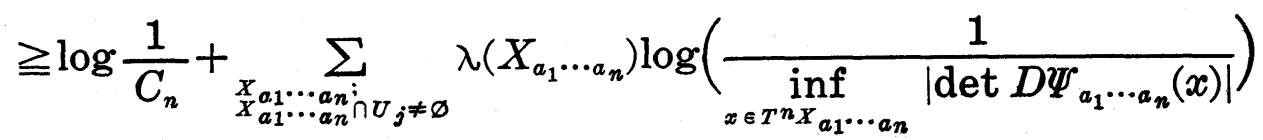

$$
\begin{aligned}
& +\sum_{\substack{X a_{1} \cdots \cdots a_{n} ; U_{j}=\varnothing \\
a_{1} \cdots a_{n} \backslash a_{j}=\varnothing}} \lambda\left(X_{a_{1} \cdots a_{n}}\right) \log \left(\frac{1}{\inf \left|\operatorname{det} D \Psi_{a_{1} \cdots a_{n}}(x)\right|}\right)
\end{aligned}
$$

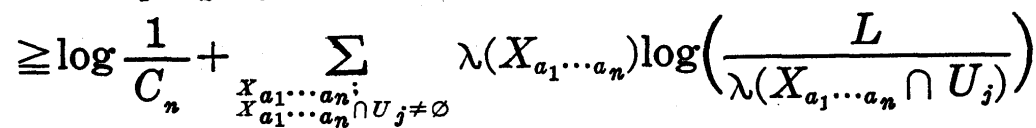

$$
\begin{aligned}
& +\sum_{\substack{X \\
X_{a_{1}} \cdots a_{n} \cap a_{n} \cap j}} \lambda\left(X_{a_{1} \cdots a_{n}}\right) \log \left(\frac{L}{\lambda\left(X_{a_{1} \cdots a_{n}}\right)}\right)
\end{aligned}
$$




$$
\begin{aligned}
& =\log \frac{L}{C_{n}}+\sum_{\substack{X \\
X_{a_{1} \cdots} \cdots a_{n} \cap a_{n} ; U_{j} \neq \varnothing}} \lambda\left(X_{a_{1} \cdots a_{n}}\right) \log \left(\frac{1}{\lambda\left(X_{a_{1} \cdots a_{n}} \cap U_{j}\right)}\right) \\
& +\sum_{\substack{X \\
X_{a_{1}} \cdots a_{n} \mathfrak{a} \backslash a_{j}=\varnothing}} \lambda\left(X_{a_{1} \cdots a_{n}}\right) \log \left(\frac{1}{\lambda\left(X_{a_{1} \cdots a_{n}}\right)}\right) \text {. }
\end{aligned}
$$

Thus

$$
\begin{aligned}
& -\sum_{\substack{X \\
X_{a_{1}} a_{1} \cdots a_{n} \cap a_{j}}} \lambda\left(X_{a_{1} \cdots a_{n}}\right) \log \lambda\left(X_{a_{1} \cdots a_{n}} \cap U_{j}\right)
\end{aligned}
$$

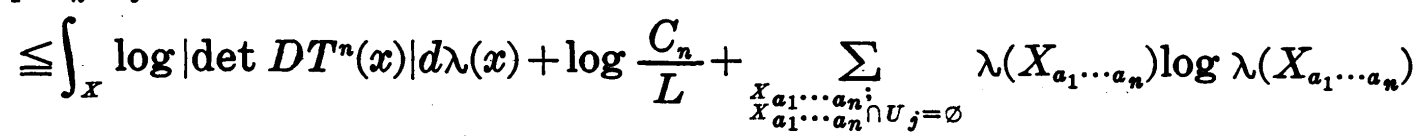

$$
\begin{aligned}
& <\int_{X} \log \left|\operatorname{det} D T^{n}(x)\right| d \lambda+\log \frac{C_{n}}{L}
\end{aligned}
$$

for all $U_{j}$. Note that $(d \mu / d \lambda) \geqq G^{-1}$ and hence

$$
\int_{X} \log \left|\operatorname{det} D T^{n}(x)\right| d \lambda \leqq G \cdot n \int_{X} \log |\operatorname{det} D T(x)| d \mu(x) .
$$

This implies the statement of (3.2-b).

From (3.1), we have

$$
\begin{aligned}
\sum_{a} \lambda\left(X_{a}\right) \log \lambda\left(X_{a}\right) & \leqq \sum_{a} \lambda\left(X_{a}\right)\left(\log G+\log \mu\left(X_{a}\right)\right) \\
& \leqq\left(\sum_{a} \lambda\left(X_{a}\right)\right) \log G+\frac{1}{M(1)} \sum \mu\left(X_{a}\right) \log \mu\left(X_{a}\right) .
\end{aligned}
$$

Therefore, (3.2-b) and the inequality

$$
-\sum_{a} \lambda\left(X_{a}\right) \log \lambda\left(X_{a}\right)+\log G \geqq-\frac{1}{M(1)} \sum \mu\left(X_{a}\right) \log \mu\left(X_{a}\right)
$$

imply (3.2-c).

From now on, using these lemmas we prove Theorem 2. Since

$$
\begin{aligned}
\frac{1}{n} \log \left\{\frac{\inf \left|\operatorname{det} D \Psi_{a_{1} \cdots a_{n}(x)}(y)\right|}{\sup _{y \in T^{n} X_{a_{1} \cdots a_{n}}}\left|\operatorname{det} D \Psi_{a_{1} \cdots a_{n}(x)}(y)\right|}\right\} & \leqq \frac{1}{n} \log \left\{\frac{\left|\operatorname{det} D \Psi_{a_{1} \cdots a_{n}(x)}\left(T^{n} x\right)\right|}{\lambda\left(X_{a_{1} \cdots a_{n}(x)}\right)}\right\} \\
& \leqq \frac{1}{n} \log \left\{\frac{\sup \left|\operatorname{det} D \Psi_{a_{1} \cdots a_{n}(x)}(y)\right|}{L \inf _{y \in T^{n} X_{a_{1} \cdots a_{n}}}\left|\operatorname{det} D \Psi_{a_{1} \cdots a_{n}(x)}(y)\right|}\right\}
\end{aligned}
$$

(where $X_{a_{1} \cdots a_{n}(x)}$ denotes a cylinder of rank $n$ containing $x$ ), by the Condition (C.7) we have 


$$
-\frac{1}{n} \log O(n) \leqq \frac{1}{n} \log \left(\frac{\left|\operatorname{det} D \Psi_{a_{1} \cdots a_{n}(x)}\left(T^{n} x\right)\right|}{\lambda\left(X_{a_{1} \cdots a_{n}(x)}\right)}\right) \leqq \frac{1}{n} \log \frac{1}{L}+\frac{1}{n} \log O(n),
$$

and hence

$$
\lim _{n \rightarrow \infty} \frac{1}{n} \log \frac{1}{\lambda\left(X_{a_{1} \cdots a_{n}(x)}\right)}=\lim _{n \rightarrow \infty} \frac{1}{n} \log \frac{1}{\left|\operatorname{det} D \Psi_{a_{1} \cdots a_{n}(x)}\left(T^{n} x\right)\right|} .
$$

Using the relation

$$
\frac{1}{\left|\operatorname{det} D \Psi_{a_{1} \cdots a_{n}(x)}\left(T^{n} x\right)\right|}=\left|\operatorname{det} D T^{n}(x)\right|,
$$

we obtain

$$
\lim _{n \rightarrow \infty} \frac{1}{\lambda\left(X_{a_{1} \cdots a_{n}(x)}\right)}=\lim _{n \rightarrow \infty} \frac{1}{n} \log \left|\operatorname{det} D T^{n}(x)\right| \text { for a.e. } x,
$$

therefore by the ergodic theorem

$$
\lim _{n \rightarrow \infty} \frac{1}{\lambda\left(X_{a_{1} \cdots a_{n}(x)}\right)}=\int_{X} \log |\operatorname{det} D T(x)| d \mu(x) \text { for a.e. } x .
$$

We remark that Lemma 3.1 allows us to have

$$
\lim _{n \rightarrow \infty} \frac{1}{n} \log \frac{\mu\left(X_{a_{1} \cdots a_{n}(x)}\right)}{\lambda\left(X_{a_{1} \cdots a_{n}(x)}\right)}=0 \text { for a.e. } x \in X .
$$

This implies

$$
\lim _{n \rightarrow \infty} \frac{1}{n} \log \frac{1}{\mu\left(X_{a_{1} \cdots a_{n}(x)}\right)}=\int_{X} \log |\operatorname{det} D T(x)| d \mu(x) \quad \text { for a.e. } x \in X .
$$

On the other hand, by Lemma 3.2, the Shannon-McMillan theorem ([1], [3]) allows us to conclude

$$
\lim _{n \rightarrow \infty} \frac{1}{n} \log \frac{1}{\mu\left(X_{a_{1} \cdots a_{n}(x)}\right)}=h(T) \quad \text { for a.e. } x \in X .
$$

Therefore we have

$$
h(T)=\int_{X} \log |\operatorname{det} D T(x)| d \mu(x) .
$$

\section{§4. Proof of Theorem 3.}

In order to prove the main theorem, we construct an invertible 
extension of $T$ as follows; let $Z$ be the set of sequences of $I, z=$ $\left(z_{-1}, z_{-2}, \cdots, z_{-n}, \cdots\right)$. We call the system $(Y, T)$ the extension of $(X, T)$, where $Y$ is the subset of $X \times Z$ composed of all pairs $(x ; z)$ such that, for all $n>0$, there exists $x_{-n}$ in $X$ satisfying

$$
T x_{-1}=x, \quad T x_{-n}=x_{-n+1}, \quad x_{-n} \in X_{s_{-n}},
$$

and $\bar{T}$ is defined by

$$
\bar{T}(x ; z)=\left(x^{\prime} ; z^{\prime}\right), \quad \text { with } \quad x^{\prime}=T x, \quad z_{-n}^{\prime}=z_{-n+1}
$$

for all $n>1$ and $z_{-1}^{\prime}$ is the unique index for which $x \in X_{s_{-1}^{\prime}}^{\prime}$.

It is easy to see that the projection $\pi$ onto $X$ commutes with the map $T$ and that, for any invariant measure $\mu$ on $X$, there exists a unique invariant measure $\bar{\mu}$ on $Y$ whose image by $\pi$ is $\mu$. If the measure $\mu$ is ergodic, so is $\bar{\mu}$. Many basic results about the natural extension of an endomorphism were stated in Rohlin's paper [13].

Now, we prepare some notations. Let $\bar{X}_{a}=\pi^{-1} X_{a}, \bar{Q}=\left\{\bar{X}_{a}\right\}, \xi=$ $\vee_{i=1}^{\infty} \bar{T}^{i} \bar{Q}$, and $\eta=\bar{Q}^{\vee} \xi$. Then, we prove a property for conditional measures in the Rohlin decomposition, with respect to $\eta$, from which weak Bernoulli property for $T$ follows. Throughout this section, we suppose the assumptions of Theorem 3 are valid.

LEMмA 4.1. The measurable partition $\eta=\bar{Q}^{\vee} \xi$ has the following properties:

$$
\begin{array}{ll}
(4.1-\mathrm{a}) & \bar{T}^{-1} \eta \geqq \eta, \\
\text { (4.1-b) } & \vee_{n=-\infty}^{\infty} \bar{T}^{-n} \eta=\varepsilon, \\
\text { (4.1-c) } & \bigwedge_{n=-\infty}^{\infty} \bar{T}^{-n} \eta=\nu, \\
\text { (4.1-d) } & h(\bar{T})=H\left(\bar{T}^{-1} \eta \mid \eta\right) .
\end{array}
$$

Proof. From the definition of $\eta$ it is immediate that $\eta$ is a measurable partition, and (4.1-a) and (4.1-b) hold. By Theorem 1, we know that $\bar{T}$ is a Kolmogorov automorphism. From this and (3.2-c) of Lemma 3.2, we have (4.1-c). (4.1-d) is an immediate consequence of (4.1-b).

Let

$$
H_{1}=\min _{X_{a} \in \mathscr{O}_{1}}\left\{\frac{\lambda\left(X_{a}\right)}{C_{1}}\right\} \quad \text { and } \quad H_{2}=\frac{1}{\inf _{x \in X}|\operatorname{det} D T(x)|} .
$$

Then we have, for any $X_{a} \in \mathscr{D}_{1}$,

$$
H_{1} \leqq\left|\operatorname{det} D \Psi_{a}(x)\right| \leqq H_{2}, \quad x \in T X_{a} .
$$


Define for any $y=(x ; z)$ and $y^{\prime}=\left(x^{\prime} ; z\right)$

$$
\Delta\left(y, y^{\prime}\right) \equiv \limsup _{j \rightarrow \infty} \prod_{n=1}^{j}\left|\frac{\operatorname{det} D T\left(\pi\left(\bar{T}^{-n} y\right)\right)}{\operatorname{det} D T\left(\pi\left(\bar{T}^{-n} y^{\prime}\right)\right)}\right| .
$$

Let

$$
\begin{aligned}
\mathscr{F}^{(n)} & \equiv\left\{\left(a_{-1}, a_{-2}, \cdots a_{-n}\right): X_{a_{-n}, a_{-n+1} \cdots a_{-1}} \in \mathscr{D}_{n}\right\}, \\
\alpha^{(n)} & \equiv\left\{\left(a_{-1} \cdots a_{-n}\right):\left(a_{-1} \cdots a_{-n+1}\right) \in \mathscr{F}^{(n-1)},\left(a_{-1} \cdots a_{-n}\right) \notin \mathscr{F}^{(n)}\right\}, \\
F^{n} & \equiv{ }_{\left(a_{-1} \cdots a_{-n}\right) \in \mathscr{F}(n)}\left(\bar{T} \bar{X}_{a_{-1}} \cap \bar{T}^{2} \bar{X}_{a_{-2}} \cap \cdots \cap \bar{T}^{n} \bar{X}_{a_{-n}}\right), \\
A^{n} & =\bigcup_{\left(a_{-1} \cdots a_{-n}\right) \in \alpha(n)}\left(\bar{T} \bar{X}_{a_{-1}} \cap \cdots \cap \bar{T}^{n} \bar{X}_{a_{-n}}\right) .
\end{aligned}
$$

We note that, from the Condition (C.3), $\left(a_{-1} \cdots a_{-n}\right) \in \mathscr{F}^{(n)}$ implies $\left(a_{-1} \cdots a_{-k}\right) \in \mathscr{F}^{(k)}$ for all $k(1 \leqq k \leqq n)$. With the above definitions, we show the following lemmas.

LEMMA 4.2. If $\left(a_{-1} \cdots a_{-n}\right) \in \alpha^{(n)}$, then there exists an integer $i(0 \leqq i \leqq$ $\left.k_{0}-1\right)$ such that $X_{a_{-n} a_{-n+1} \cdots a_{-n+i}} \in R(C . T)$.

Proof. If $n \leqq k_{0}$, then it is trivial. Let $n>k_{0}$, and suppose that for all $i\left(0 \leqq i \leqq k_{0}-1\right)$

$$
X_{a_{-n} \cdots a_{-n+i}} \notin R(C . T) \text {. }
$$

Then we have $X_{a_{-n} \cdots a_{-n+k 0-1}} \in \mathscr{D}_{k_{0}}$. On the other hand, from (C.9) if $X_{a_{-n+1} \cdots a_{-1}} \in \mathscr{D}_{n-1}$ and $X_{a_{-n} \cdots a_{-1}} \in \mathscr{D}_{n}^{c}$, then

$$
X_{a_{-n} \cdots a_{-1}} \subset \bigcup_{j=1}^{k_{0}} B_{j} .
$$

This contradicts (4.2.1).

LemmA 4.3. $\lim _{n \rightarrow \infty} \bar{\mu}\left(F^{n}\right)=0$, and therefore $Y=\cup_{n=1}^{\infty} A^{n}(\bar{\mu} \bmod 0)$.

Proof. We note that $\bar{\mu}\left(F^{n}\right)=\mu\left(D_{n}\right)$. From (C.4) and relations

$$
\begin{aligned}
\mu\left(D_{n}\right) & =\sum_{m=0}^{\infty}\left(\sum_{X_{k(m) \in \mathscr{D}_{m}}}\left(\sum_{\left.\left.X_{a(n) \in \mathscr{C}_{n}} \nu\left(X_{k(m) a(n)}\right)\right)\right)}\left|\operatorname{det} D \Psi_{k(m)}(x)\right| d \lambda(x)\right) \cdot G,\right. \\
& \leqq \sum_{m=0}^{\infty}\left(\sum_{x_{k(m)} \in \mathscr{S}_{m}} \int_{D_{n} \cap T^{m} X_{k(m)}} \mid\right.
\end{aligned}
$$

we have $\lim _{n \rightarrow \infty} \bar{\mu}\left(F^{n}\right)=0$. (Here $k(m)=\left(k_{1} \cdots k_{m}\right)$, and $\left.a(n)=\left(a_{1} \cdots a_{n}\right)\right)$. And hence, the equality 


$$
\bar{\mu}\left(\left(\bigcup_{n=1}^{\infty} A^{n}\right)^{0}\right)=\mu\left(\left(\bigcup_{n=1}^{\infty} B_{n}\right)^{\circ}\right)
$$

implies the second assertion.

For each $k$ with $0 \leqq k \leqq k_{0}-1$, we define for $n>k$

$$
\alpha_{k}^{(n)}=\left\{\left(a_{-1} \cdots a_{-n}\right) \in \alpha^{(n)}: X_{a_{-1} \cdots a_{-n+k}} \in R(C . T)\right\},
$$

and

$$
A_{k}^{n}=\bigcup_{\left(a_{-1} \ldots a_{-n}\right) \in \alpha_{k}^{(n)}}\left(\bar{T} \bar{X}_{a_{-1}} \cap \cdots \cap \bar{T}^{n} \bar{X}_{a_{-n}}\right) .
$$

Then by Lemma 4.2 we can easily see that

$$
\text { for } n>k_{0} \alpha^{(n)}=\bigcup_{k=0}^{k_{0}-1} \alpha_{k}^{(n)}, \quad A^{n}=\bigcup_{k=0}^{k_{0}-1} A_{k}^{n}
$$

and

$$
\text { for } n \leqq k_{0} \alpha^{(n)}=\bigcup_{k=0}^{n-1} \alpha_{k}^{(n)}, \quad A^{n}=\bigcup_{k=0}^{n-1} A_{k}^{n},
$$

where the above unions are disjoint.

LEMmA 4.4. For $\bar{\mu}$ a.e. $y \in Y$, there exists a positive integer $K=K(y)$ such that

$$
\begin{aligned}
& \text { for any } y^{\prime} \in \xi(y) \text { we have } \\
& \qquad \frac{1}{C} \cdot\left(\frac{H_{1}}{H_{2}}\right)^{K(y)} \leqq \Delta\left(y, y^{\prime}\right) \leqq C \cdot\left(\frac{H_{2}}{H_{1}}\right)^{K(y)},
\end{aligned}
$$

and so

$$
\begin{aligned}
& \frac{1}{C}\left(\frac{H_{1}}{H_{2}}\right)^{K(y)} \int_{\eta(y)} d y^{\prime} \leqq \int_{\eta(y)} \Delta\left(y, y^{\prime}\right) d y^{\prime} \\
& \leqq C\left(\frac{H_{2}}{H_{1}}\right)^{K(y)} \int_{\eta(y)} d y^{\prime},
\end{aligned}
$$

where $\xi(y)$ and $\eta(y)$ denote the elements of $\xi$ and $\eta$ containing $y$ respectively, and $d$ denotes the natural Lebesgue measure on each element of $\eta$.

Proof. For $y \in A_{k}^{n}$ from (C.3) and (4.1) we can easily see that for any $y^{\prime} \in \xi(y)$

$$
\frac{1}{C}\left(\frac{H_{1}}{H_{2}}\right)^{n-k-1} \leqq \Delta\left(y, y^{\prime}\right) \leqq C\left(\frac{H_{2}}{H_{1}}\right)^{n-k-1} .
$$

Putting $K(y)=n-k-1$, we have the statement of Lemma 4.4 immediately. 
As we have announced in introduction, our main goal of this section is to prove

Proposition 4.5. The conditional measures of $\bar{\mu}$ with respect to the partition $\eta$ are given by

$$
q(y, B)=\frac{\int_{B \cap \eta(y)} \Delta\left(y, y^{\prime}\right) d y^{\prime}}{\int_{\eta(y)} \Delta\left(y, y^{\prime}\right) d y^{\prime}}
$$

for each $y \in Y$ and $B$ a measurable subset.

To prove this proposition, we have to show the following:

LEMMA 4.6. For each $n>0, \log q\left(y,\left[\bar{T}^{-n} \eta\right](y)\right)$ is $\bar{\mu}$-integrable.

REMARK 5. With this done, we can prove Proposition 4.5 as Ledrappier did in [6]. Therefore, we only give the outline of the argument used for this part. In fact, since the following equality is valid:

$\log q\left(y,\left[\bar{T}^{-n} \eta\right](y)\right)=-\log \left|\operatorname{det} D T^{n}(\pi y)\right|+\log \left\{k \circ \bar{T}^{n}(y)\right\}-\log k(y)$,

where $k(y)=\int_{\eta(y)} \Delta\left(y, y^{\prime}\right) d y^{\prime}$, Lemma 4.6 allows us to apply the next classical lemma to our case;

Lemma (cf. [6]). Let $(Y, \bar{\mu}, \bar{T})$ be a dynamical system and $g_{i}, i=$ $1,2,3$, be functions related by $g_{1}=g_{2}+g_{3} \circ \bar{T}-g_{3}$, with $g_{2}$ and $g_{1}$ integrable. Then we have

$$
\lim \frac{1}{n} g_{3} \circ \bar{T}^{n}=\widehat{g}_{1}-\widehat{g}_{2}=0 \quad \bar{\mu} \text { a.e. , }
$$

where $\hat{g}$ denotes the point-wise limit of ergodic averages of $g$. Then we can see that the following relation is true:

$$
\begin{aligned}
-\int \log q\left(y,\left[\bar{T}^{-n} \eta\right](y)\right) d \bar{\mu}(y) & =\int \log \left|\operatorname{det} D T^{n}(x)\right| d \mu(x) \\
& =n \int \log |\operatorname{det} D T(x)| d \mu(x) .
\end{aligned}
$$

On the other hand, if $p(y, \cdot)$ is the conditional measure of $\bar{\mu}$ with respect to $\eta$, then by Lemma 4.1, (4.1-d) we have

$$
n \cdot h(T)=H\left(\bar{T}^{-n} \eta \mid \eta\right)=-\int \log p\left(y,\left[\bar{T}^{-n} \eta\right](y)\right) d \bar{\mu}(y) .
$$


Therefore, by the Rohlin's formula we obtain

$$
\int \log \frac{q\left(y,\left[\bar{T}^{-n} \eta\right](y)\right)}{p\left(y,\left[\bar{T}^{-n} \eta\right](y)\right)} d \bar{\mu}(y)=0 .
$$

By the concavity of the function $\log$ and from (4.1-b), the proposition follows by letting $n$ go to infinity (cf. [7], [11]).

Proof of Lemma 4.6. Let $y \in A_{k}^{\pi}, y=\left(x ; a_{-1}, a_{-2}, \cdots a_{-m+k+1}, a_{-m+k} \cdots\right.$ $\left.a_{-m} \cdots\right)$. Then a simple calculation gives

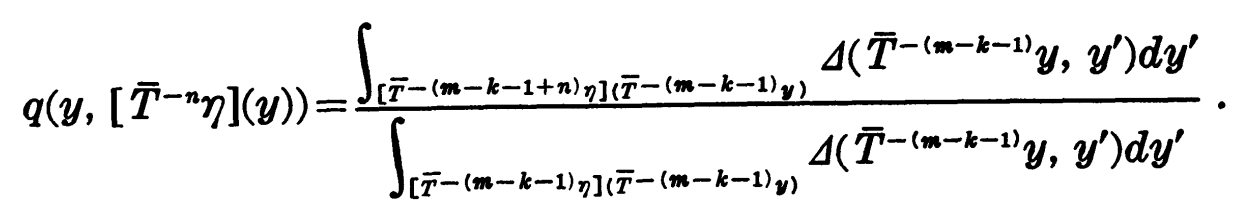

Since $(1 / C) \leqq \Delta\left(\bar{T}^{-(m-k-1)} y, y^{\prime}\right) \leqq C$ on $\eta\left(\bar{T}^{-(m-k-1)} y\right)$, this implies the following:

$$
\begin{aligned}
& q\left(y,\left[\bar{T}^{-n} \eta\right](y)\right) \geqq \frac{1}{C^{2}} \cdot \frac{\int_{[\bar{T}-(m-k-1+n) \eta](\bar{T}-(m-k-1)} d y^{\prime}}{\left.\int_{[\bar{T}-(m-k-1) \eta]} d \bar{T}^{\prime}-(m-k-1) y\right)} \\
& =\frac{1}{C^{2}} \cdot \frac{\left.\lambda\left(X_{a_{-m+k+1} \cdots a_{0} a_{1} \cdots a_{n}(\pi(\bar{T}-(m-k-1)}\right) \cap U_{j(y)}\right)}{\left.\left.\lambda\left(X_{a_{-m+k+1} \cdots a_{0}(\pi(\bar{T}-(m-k-1)}\right)\right) \cap U_{j(y)}\right)} \\
& \geqq \frac{1}{C^{2}} \cdot \frac{\left.\lambda\left(X_{a_{-m+k+1} \cdots a_{0} a_{1} \cdots a_{n}(\pi(\bar{T}-(m-k-1)}\right) \cap U_{j(y)}\right)}{\left.\lambda\left(X_{a_{-m+k+1} \cdots a_{0}(\pi(\bar{T}-(m-k-1)}\right)\right)},
\end{aligned}
$$

where $\pi\left(\bar{T}^{k} y\right) \in X_{a_{k}}(0 \leqq k \leqq n)$ and

$$
U_{j(y)} \equiv \bigcap_{j=0}^{\infty} T^{j+1} X_{a_{-m+k-j} \cdots a_{-m+k}} .
$$

Note that there exists $X_{b_{1} \cdots b_{l}}$ such that $U_{j(y)}=T^{l} X_{b_{1} \cdots b_{l}}$ and hence we can write $X_{a_{-m+k+1} \cdots a_{-1}} \cap U_{j(y)}=T^{l}\left(X_{b_{1} \cdots b_{l} a_{-m+k+1} \cdots a_{-1}}\right)$.

Then we can see that

$$
\begin{aligned}
T^{m-k-1} & \left(X_{a_{-m+k+1} \cdots a_{-1} a_{0} \cdots a_{n}} \cap U_{j(y)}\right) \\
& =T^{m-k-1}\left(T^{l} X_{b_{1} \cdots b_{l} a_{-m+k+1} \cdots a_{-1}} \cap T^{-(m-k-1)} X_{a_{0} \cdots a_{n}}\right) \\
& =U_{t(y)} \cap X_{a_{0} \cdots a_{n}(y)}
\end{aligned}
$$

for some $t(y) \in\{0,1, \cdots, N\}$. Using this, we have

$$
\begin{aligned}
& \lambda\left(X_{a_{-m+k+1} \cdots a_{-1} a_{0} \cdots a_{n}} \cap U_{j(y)}\right) \\
& \quad=\lambda\left(\Psi_{a_{-m+k+1} \cdots a_{-1}} \circ T^{m-k-1}\left(X_{a_{-m+k+1} \cdots a_{n}} \cap U_{j(y)}\right)\right) \\
& \quad=\int_{U_{t(y)} \cap X_{a_{0} \cdots a_{n}(y)}}\left|\operatorname{det} D \Psi_{a_{-m+k+1} \cdots a_{-1}}\left(x^{\prime}\right)\right| d \lambda\left(x^{\prime}\right)
\end{aligned}
$$




$$
\sum_{x^{\prime} \in U_{t(y)} \cap X_{a_{0}} \cdots a_{n}(y)}\left|\operatorname{det} D \Psi_{a_{-m+k+1} \cdots a_{-1}}\left(x^{\prime}\right)\right| \cdot \lambda\left(U_{t(y)} \cap X_{a_{0} \cdots a_{n}(y)}\right) .
$$

On the other hand, the following inequality is true:

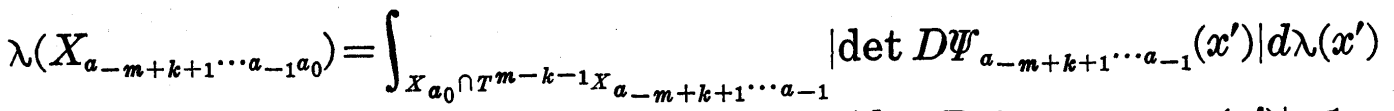

$$
\begin{aligned}
& \leqq_{x^{\prime} \in X_{a_{0}} \cap T^{m-k}-1_{X^{\prime}}} \sup _{a_{-m+k+1} \cdots a_{-1}}\left|\operatorname{det} D \Psi_{a_{-m+k+1} \cdots a_{-1}}\left(x^{\prime}\right)\right| \cdot 1 \\
& \leqq_{x^{\prime} \in T^{m-k-1} X_{a_{-m+k+1} \cdots a_{-1}}}\left|\operatorname{det} D \Psi_{a_{-m+k+1} \cdots a_{-1}}\left(x^{\prime}\right)\right| \cdot
\end{aligned}
$$

Combining the above results, we obtain

$$
\begin{aligned}
& q\left(y,\left[\bar{T}^{-n} \eta\right](y)\right) \\
& \geqq \frac{1}{C^{2}} \frac{\inf _{x^{\prime} \in T^{m-k-1} X_{a_{-m+k+1} \cdots a_{-1}}}\left|\operatorname{det} D \psi_{a_{-m+k+1} \cdots a_{-1}}\left(x^{\prime}\right)\right|}{\left|\operatorname{det} D \psi_{a_{-m+k+1} \cdots a_{-1}}\left(x^{\prime}\right)\right|} \lambda\left(U_{t(y)} \cap X_{a_{0} \cdots a_{n}(y)}\right),
\end{aligned}
$$

and therefore by the Condition (C.7)

$$
q\left(y,\left[\bar{T}^{-n} \eta\right](y)\right) \geqq\left\{\begin{array}{l}
\frac{1}{C^{2}} \lambda\left(X_{a_{0} \cdots a_{n}(y)} \cap U_{t(y)}\right) \quad \text { if } y \in A_{0}^{1} \\
\frac{1}{C^{2}} \lambda\left(X_{a_{0} \cdots a_{n}(y)} \cap U_{t(y)}\right) \cdot O\left(\frac{1}{(m-k-1)^{l}}\right) \\
\geqq \frac{1}{C^{2}} \lambda\left(X_{a_{0} \cdots a_{n}(y)} \cap U_{t(y)}\right) \cdot O\left(\frac{1}{(m-1)^{l}}\right) \quad \text { if } y \in A_{k}^{m}(m \geqq 2) .
\end{array}\right.
$$

From this and the equality

$$
\int_{Y} \log q\left(y,\left[\bar{T}^{-n} \eta\right](y)\right) d \bar{\mu}(y)=\sum_{m=1}^{\infty} \int_{A^{m}} \log q\left(y,\left[\bar{T}^{-n} \eta\right](y)\right) d \bar{\mu}(y),
$$

it follows that

$$
\begin{aligned}
\int_{Y} \log & q\left(y,\left[\bar{T}^{-n} \eta\right](y)\right) d \bar{\mu}(y) \\
\geqq & \int_{A_{0}^{1}}\left\{\log \frac{1}{C^{2}}+\log \lambda\left(X_{a_{0} \cdots a_{n}(y)} \cap U_{t(y)}\right)\right\} d \bar{\mu}(y) \\
& +\sum_{m=2}^{\infty} \int_{A^{m}}\left\{\log \frac{1}{C^{2}}+\log \lambda\left(X_{a_{0} \cdots a_{n}(y)} \cap U_{t(y)}\right)+\log O\left(\frac{1}{(m-1)^{l}}\right)\right\} d \bar{\mu}(y) \\
\geqq & 2\left\{\left(\log \frac{1}{C^{2}}\right) \times \bar{\mu}(Y)+\int_{Y} \log \lambda\left(X_{a_{0} \cdots a_{n}(y)} \cap U_{t(y)}\right) d \bar{\mu}(y)\right\} \\
& +\sum_{m=2}^{\infty} \bar{\mu}\left(A^{m}\right) \log \left(O\left(\frac{1}{(m-1)^{l}}\right)\right) .
\end{aligned}
$$


Now, we estimate $\int_{Y} \log \lambda\left(X_{a_{0} \cdots a_{n}(y)} \cap U_{t(y)}\right) d \bar{\mu}(y)$. For each $i \epsilon$ $\{0, \cdots, N\}$, let $\mathscr{U}_{i}=\left\{y \in Y: U_{t(y)}=U_{i}\right\}$, where

$$
U_{t(y)}=T^{m-k-1}\left(U_{j(\bar{T}-m+k+1} \cap \cap X_{a_{-m+k+1} \cdots a_{-1}}\right) \text { for } y \in A_{k}^{m} \text {. }
$$

Then we have

$$
\begin{aligned}
\int_{Y} \log & \lambda\left(X_{a_{0} \cdots a_{n}(y)} \cap U_{t(y)}\right) d \bar{\mu}(y) \\
& =\sum_{i=0}^{N} \int_{\mathscr{K}_{i}} \log \lambda\left(X_{a_{0} \cdots a_{n}(y)} \cap U_{i}\right) d \bar{\mu}(y) \\
& \geqq \sum_{i=0}^{N} \int_{Y} \log \lambda\left(X_{a_{0} \cdots a_{n}(y)} \cap U_{i}\right) d \bar{\mu}(y) \\
& =\sum_{i=0}^{N}\left({ }_{\left(a_{0} \cdots a_{n}\right) \in \Delta(n)} \mu\left(X_{a_{0} \cdots a_{n}}\right) \log \lambda\left(X_{a_{0} \cdots a_{n}} \cap U_{i}\right)\right) \\
& \geqq M(n+1) \sum_{i=0}^{N}\left({ }_{\left(a_{0} \cdots a_{n}\right) \in \Delta(n)} \lambda\left(X_{a_{0} \cdots a_{n}}\right) \log \lambda\left(X_{a_{0} \cdots a_{n}} \cap U_{i}\right)\right),
\end{aligned}
$$

(the last inequality follows from Lemma 3.1). Therefore by (3.2-b) of Lemma 3.2 $\int_{Y} \log \lambda\left(X_{a_{0} \cdots a_{n}(y)} \cap U_{t(y)}\right) d \bar{\mu}(y)>-\infty$. obtain

Next, we estimate $\sum_{m=2}^{\infty} \bar{\mu}\left(A^{m}\right) \log \left(1 / O\left\{(m-1)^{l}\right\}\right)$. By Lemma 3.1, we

$$
\begin{aligned}
\bar{\mu}\left(A^{m}\right) & =\sum_{\left(a_{-1} \cdots a_{-m}\right) \in \alpha(m)} \mu\left(X_{a_{-m} \cdots a_{-1}}\right) \\
& \leqq \hat{M}\left(k_{0}\right) \sum_{\left(a_{-1} \cdots a_{-m}\right) \in \alpha(m)} \lambda\left(X_{a_{-m} \cdots a_{-1}}\right) \\
& =\hat{M}\left(k_{0}\right)\left\{\sum_{\left(a_{-1} \cdots a_{-m}\right) \in \alpha(m)} \int_{T X_{a_{-}-m} \cap X_{a_{-m+1} \cdots a_{-1}}}\left|\operatorname{det} D \Psi_{a_{-m}}(x)\right| d \lambda(x)\right\}
\end{aligned}
$$

and by (C.7)

$$
\begin{aligned}
& \bar{\mu}\left(A^{m}\right) \leqq \hat{M}\left(k_{0}\right)\left\{\sum_{\left(a_{-1} \cdots a_{-m}\right) \in \alpha^{(m)}} C_{1} \cdot \inf _{\boldsymbol{x} \in T X_{a_{-m}}}\left|\operatorname{det} D \Psi_{a_{-m}}(x)\right| \cdot \lambda\left(X_{a_{-m+1} \cdots a_{-1}}\right)\right\} \\
& \leqq \hat{M}\left(k_{0}\right) \cdot C_{1}\left\{\sum_{\left(a_{-1} \cdots a_{-m}\right) \in \alpha(m)} \int_{T X_{a-m}}\left|\operatorname{det} D \Psi_{a_{-m}}(x)\right| d \lambda \cdot \frac{1}{L} \lambda\left(X_{a_{-m+1} \cdots a_{-1}}\right)\right\}
\end{aligned}
$$

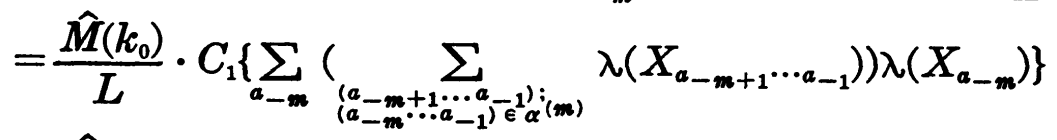

$$
\begin{aligned}
& \leqq \frac{\hat{M}\left(k_{0}\right) C_{1}}{L} \cdot \lambda\left(D_{m-1}\right)\left\{\sum_{a_{-m}} \lambda\left(X_{a_{-m}}\right)\right\} \\
& \leqq \frac{\hat{M}\left(k_{0}\right)}{L} \cdot C_{1} \cdot \lambda\left(D_{m-1}\right) \text {. }
\end{aligned}
$$

Therefore by $(\mathrm{C} .4)^{*}$ we have $\sum_{m=2}^{\infty} \bar{\mu}\left(A^{m}\right) \log \left\{1 / O\left((m-1)^{l}\right)\right\}>-\infty$. Conse- 
quently we have the conclusion of Lemma 4.6.

Proposition 4.5 implies that the conditional measures of $\bar{\mu}$ with respect to $\eta$ are all absolutely continuous with respect to $\bar{\mu}$. Note that by Theorem $2 \bar{T}$ is a Kolmogorov automorphism and hence the Pinsker partition $\wedge_{n=-\infty}^{\infty} \vee_{k=-\infty}^{n} \bar{T}^{k} \bar{Q}$ is trivial. Combining these results, we see that the sufficient condition for the weak Bernoulli partition is satisfied, i.e. the conditional measures of $\bar{\mu}$ with respect to $\eta$ must all coincide on the remote past $\sigma$ algebra $\sigma\left(\wedge_{n=-\infty}^{\infty} \vee_{k=-\infty}^{-n} \bar{T}^{k} \bar{Q}\right)$, and this property of the conditional measures implies that $Q$ is a weak Bernoulli partition. More detailed proof may be found for example in [6] [12] (cf. [8] [10]).

\section{§5. Examples.}

In this section, we present three examples to which we can apply our theorem. First we consider the following one parameter family of maps on an interval.

EXAmple 1. Let $X=[0,1]$, and for $\alpha$ with $0<\alpha<1$ define

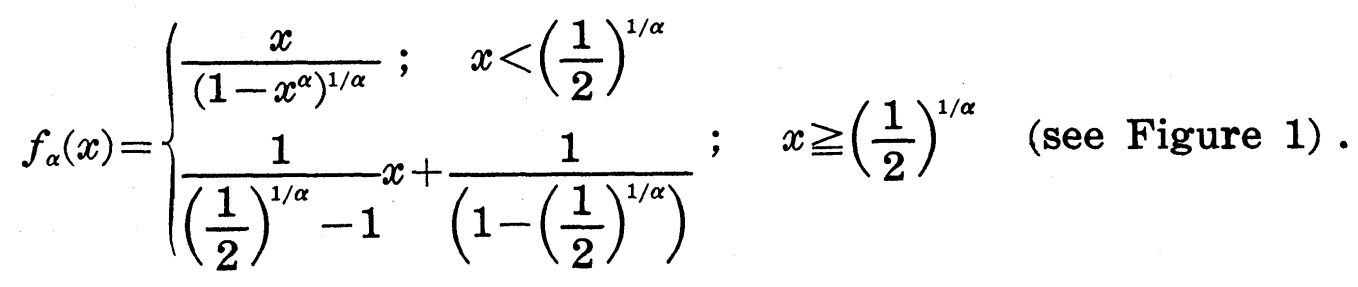

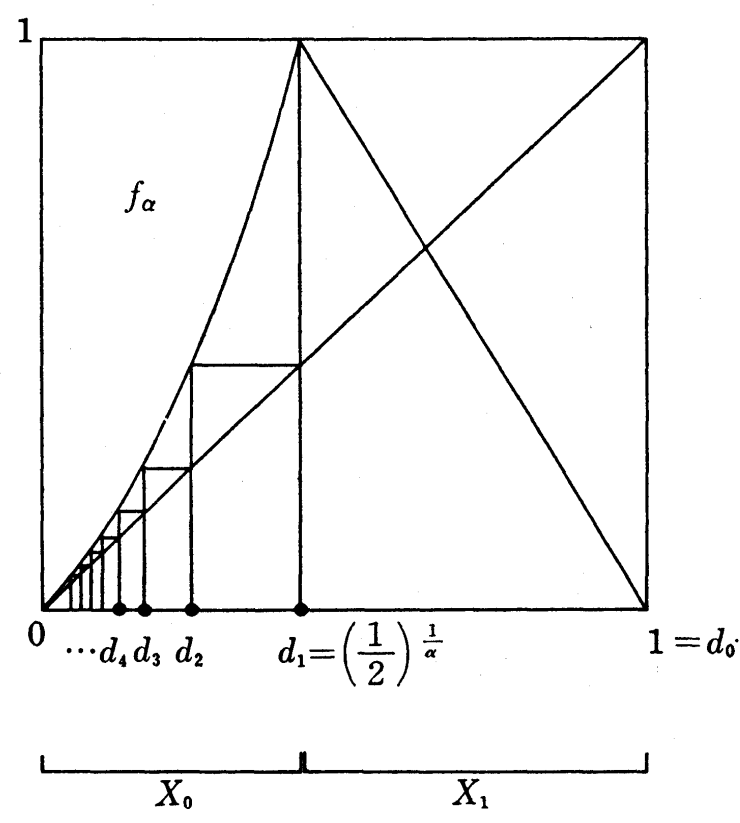

Figure 1 
Let $X_{0}=\left[0,(1 / 2)^{1 / \alpha}\right), \quad X_{1}=\left[(1 / 2)^{1 / \alpha}, 1\right], \quad \Psi_{0}(x)=x /\left(\left(1+x^{\alpha}\right)^{1 / \alpha}\right), \quad \Psi_{1}(x)=\left\{(1 / 2)^{1 / \alpha}-\right.$ $1\} x+1$. Then simple calculation gives the following properties:

(1) $\left(\Psi_{0}\right)^{\prime}(x)$ is a positive and monotone decreasing function on $[0,1]$,

(2) $\left(\left(\Psi_{0}\right)^{\prime \prime} /\left(\Psi_{0}\right)^{\prime}\right)(x)$ is a negative and monotone increasing function on $[0,1]$. Let $\underbrace{X_{00 \cdots 01}}_{n}=\left[d_{n}, d_{n-1}\right)$. From (1), we have

$$
\Psi_{0}^{\prime}\left(d_{k-1}\right) \leqq \Psi_{0}^{\prime}(x) \leqq \Psi_{0}^{\prime}\left(d_{k}\right) \quad \text { for any } \quad x \in \underbrace{X_{00} \cdots 0_{1}}_{i} .
$$

Therefore

$$
\frac{\sup _{x \in X}\left|\left(\Psi_{00 \cdots 01}\right)^{\prime}(x)\right|}{\inf _{x \in X}\left|\left(\Psi_{n}^{00 \cdots 01}\right)^{\prime}(x)\right|} \leqq \frac{\Psi_{0}^{\prime}\left(d_{n}\right)}{\Psi_{0}^{\prime}(1)} \leqq 2^{1+1 / \alpha} .
$$

We remark that

(4) if

$$
n \geqq\left[\frac{2\left(2^{1 / \alpha}-1\right)^{\alpha /(\alpha+1)}-2^{1 /(\alpha+1)}}{2^{1 /(\alpha+1)}-\left(2^{1 / \alpha}-1\right)^{\alpha /(\alpha+1)}}+1\right]
$$

(where [ ] denotes the integer part of a number), then

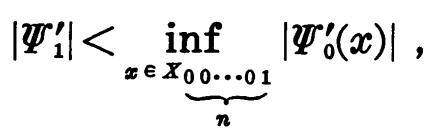

and

(5) for any sequence $\left(a_{1} \cdots a_{n}\right) \in A(n)$ such that $a_{k}=1$ for some $k \in$ $\{1,2, \cdots, n-1\}$, we have $d_{n-1} \leqq \inf _{x \in X_{a_{1} \cdots a_{n}}}\{x\}$.

From the above properties, we can show that there exists a constant $C>1$ such that for all $n>0$ and any $\left(a_{1} \cdots a_{n}\right) \in A(n)$ with $a_{n}=1$

$$
\frac{\sup _{x \in X}\left|\left(\Psi_{a_{1} \cdots a_{n}}\right)^{\prime}(x)\right|}{\inf _{x \in X}\left|\left(\Psi_{a_{1} \cdots a_{n}}\right)^{\prime}(x)\right|}<C \text {. }
$$

In fact, by the mean value theorem for any $\theta, \theta^{\prime} \in[0,1]$ such that $\theta^{\prime}-\theta>0$, we have

(6)

$$
\log \left|\frac{\left(\Psi_{00 \cdots 01}\right)^{\prime}\left(\theta^{\prime}\right)}{\left(\Psi_{n}^{00 \cdots 01}\right)^{\prime}(\theta)}\right|=\sum_{j=1}^{n-1}\left|\left(\frac{\Psi_{0}^{\prime \prime}}{\Psi_{0}^{\prime}}\right)\left(\xi_{j}\left(\theta, \theta^{\prime}\right)\right)\right| \mid\left(\Psi_{\underbrace{0 \cdots 0_{1}}_{0-j}}\right)^{\prime}\left(\eta_{j}\left(\theta, \theta^{\prime}\right) \mid \cdot\left(\theta^{\prime}-\theta\right)\right.
$$

where $\xi_{j}\left(\theta, \theta^{\prime}\right) \in \underbrace{X_{00 \cdots 01}}_{n-j}, \eta_{j}\left(\theta, \theta^{\prime}\right) \in X$. 
On the other hand, for any $x, x^{\prime} \in[0,1]$ Properties (1), (2), (3), (4), and (5) allow us to have the following:

( 7 )

$$
\begin{aligned}
& |\log | \frac{\left(\Psi_{a_{1} \cdots a_{n}}\right)^{\prime}\left(x^{\prime}\right)}{\left(\Psi_{a_{1} \cdots a_{n}}\right)^{\prime}(x)}|| \leqq \sum_{j=1}^{n-1}\left|\left(\frac{\Psi_{a_{j}}^{\prime \prime}}{\Psi_{a_{j}}^{\prime}}\right)\left(\xi_{j}\left(x, x^{\prime}\right)\right)\right| \cdot\left|\left(\Psi_{a_{j+1} \cdots a_{n}}\right)^{\prime}\left(\eta_{j}\left(x, x^{\prime}\right)\right)\right| \cdot\left|x^{\prime}-x\right| \\
& \leqq \sum_{j=1}^{n-1} \inf _{x \in X_{0} \underbrace{}_{n-j} \ldots 101}\left|\frac{\Psi_{0}^{\prime \prime}}{\Psi_{0}^{\prime}}(x)\right|_{x \in X_{X_{0} 0 \cdots 01} \inf _{n-j-1}} \Psi_{0}^{\prime}(x) \cdots \inf _{x \in X} \Psi_{0}^{\prime}(x)\left(\frac{\left|\Psi_{1}^{\prime}\right|}{\Psi_{0}^{\prime}(1)}\right)^{n_{0}},
\end{aligned}
$$

where

$$
n_{0}=\left[\frac{2\left(2^{1 / \alpha}-1\right)^{\alpha /(\alpha+1)}-2^{1 /(\alpha+1)}}{2^{1 /(\alpha+1)}-\left(2^{1 / \alpha}-1\right)^{\alpha /(\alpha+1)}}+1\right] .
$$

Combining (5) and (6), we can take for $C 2^{(1+1 / \alpha) \cdot 2 \cdot\left(2^{1 / \alpha}-1\right)}$. It is easy to see that (C.3) is satisfied and $B_{n}=\underbrace{X_{00 \cdots 01}}_{n}$, and $D_{n}=\underbrace{X_{00 \cdots 0}}_{n}$. To verify (C.1), we remark that for all $n \geqq n_{0}$,

$$
\lambda\left(X_{a_{1} \cdots a_{n}}\right) \leqq C\left(\frac{\left|\Psi_{1}^{\prime}\right|}{\Psi_{0}^{\prime}(1)}\right)^{n_{0}} \cdot \lambda(\underbrace{X_{00 \cdots 0}}_{n}) .
$$

In fact, for any $\left(a_{1} \cdots a_{n}\right) \in A(n)$, there exists $k$ with $0 \leqq k \leqq n$ such that $X_{a_{1} \cdots a_{k}} \in R\left(C . f_{\alpha}\right)$ and $X_{a_{k+1} \cdots a_{n}}=D_{n-k}$. Therefore

$$
\begin{aligned}
& \lambda\left(X_{a_{1} \cdots a_{n}}\right)=\int_{X_{X_{00 \cdots 0} \cdots 0}}\left|\left(\Psi_{a_{1} \cdots a_{k}}\right)^{\prime}(x)\right| d x \\
& <C \cdot \inf _{x \in \underbrace{}_{00} \underbrace{}_{n-k} \cdots 0}\left|\left(\Psi_{a_{1} \cdots a_{k}}\right)^{\prime}(x)\right| \cdot \lambda\left(X_{\frac{0}{0} \cdots 0}\right)
\end{aligned}
$$

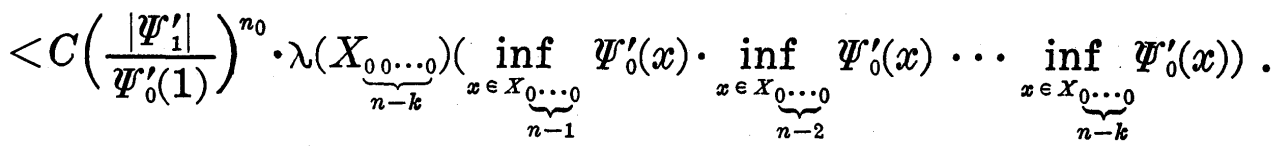

On the other hand

$$
\begin{aligned}
& \lambda\left(X_{n}^{X_{0 \ldots 0} \cdots}\right)=\int_{\underset{X_{0}^{0} \cdots 0}{n-k}}\left|\Psi_{k}^{0 \ldots 0^{0}}(x)\right| d x \\
& \geqq\left(\inf _{x \in D_{n-1}} \Psi_{0}^{\prime}(x) \cdot \inf _{x \in D_{n-2}} \Psi_{0}^{\prime}(x) \cdots \inf _{x \in D_{n-k}} \Psi_{0}^{\prime}(x)\right) \cdot \lambda\left(\underset{n=k}{X_{0} \cdots 0}\right),
\end{aligned}
$$

consequently the above assertion is valid. From this and the fact $\lim _{n \rightarrow \infty} \lambda\left(\underset{n}{X_{0.0}}\right)=0$, we have (C.1). The Conditions (C.2), (C.4)*, (C.6) and 
(C.9) are easily checked. Note that $\Psi_{0 \ldots 0}(x)=x /\left(1+m x^{\alpha}\right)^{1 / \alpha}$ and hence $\left(\Psi_{0 \ldots 0}\right)^{\prime}(x)=1 /\left(1+m x^{\alpha}\right)^{1+1 / \alpha}$. Then a direct calculation gives

$$
\begin{aligned}
W_{n} & =\sum_{m=0}^{\infty} \frac{1}{\left(1+m\left(1 /(n+1)^{1 / \alpha}\right)^{\alpha}\right)^{1+1 / \alpha}} \\
& \leqq(n+1)^{1+1 / \alpha}\left(\sum_{m=0}^{\infty} \frac{1}{(1+m)^{1+1 / \alpha}}\right),
\end{aligned}
$$

and

$$
\frac{\sup _{x \in X}\left(\Psi_{0 \ldots 0}\right)^{\prime}(x)}{\inf _{x \in X}\left(\Psi_{0 \ldots 0}\right)^{\prime}(x)} \leqq(1+m)^{1+1 / \alpha}
$$

From these, (C.5) and (C.7) are satisfied. (C.8) and (C.9) are trivial. Therefore this example satisfies all of assumptions of Theorem 3.

Next, we consider a skew product transformation which is associated to Diophantine approximation in inhomogeneous linear class.

EXAMPLE 2. Let $X=\left\{(x, y) \in R^{2}: 0 \leqq y \leqq 1,-y \leqq x<-y+1\right\}$ and define $T$ on $X$ by

$$
T(x, y)=\left(\left(\frac{1}{x}\right)-\left[\frac{1-y}{x}\right]-\left[-\frac{y}{x}\right],-\left[-\frac{y}{x}\right]-\frac{y}{x}\right) \text { (see Figure 2). }
$$

This transformation is a multi-dimensional mapping with a finite range structure and has a finite invariant measure whose density is unbounded

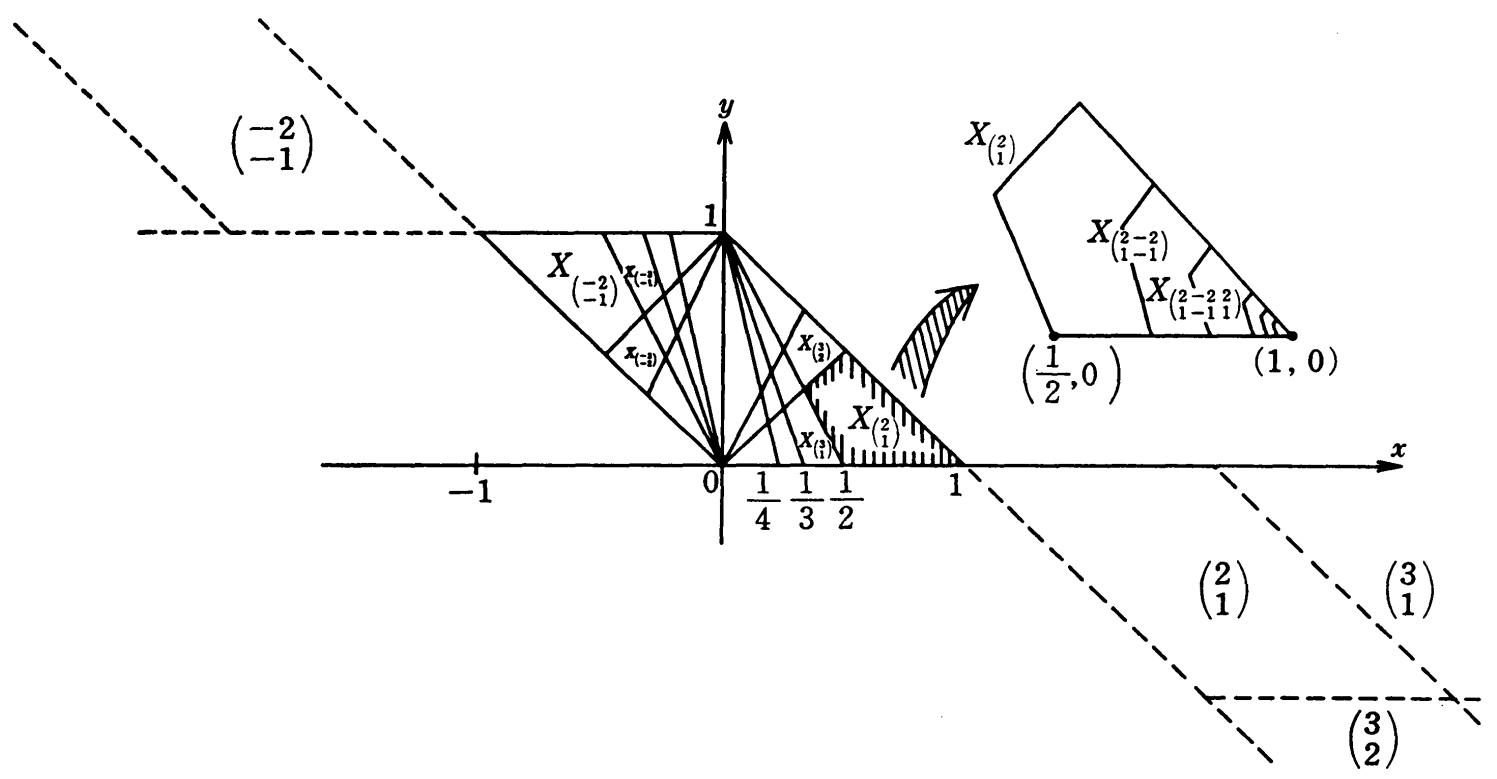

FIgURE 2 
(see [4] and [5]).

Let

$$
\begin{aligned}
& a(x, y)=\left[\frac{1-y}{x}\right]+\left[-\frac{y}{x}\right], \quad b(x, y)=-\left[-\frac{y}{x}\right], \\
& a_{k}(x, y)=a\left(T^{k-1}(x, y)\right), \quad b_{k}(x, y)=b\left(T^{k-1}(x, y)\right),
\end{aligned}
$$

and $q_{n}=a_{n} q_{n-1}+q_{n-2}\left(q_{0}=1, q_{1}=0\right)$. Then

$$
\operatorname{det} D \Psi_{\left(\begin{array}{c}
\left.a_{1} \cdots \cdots a_{n}\right) \\
b_{1} \cdots \cdots
\end{array}\right)}(x, y)=\frac{1}{\left(q_{n}+x q_{n-1}\right)^{3}} .
$$

Moreover, we can easily to see that

$$
\begin{aligned}
& \mathscr{D}_{n}=\left\{X_{\left.\left(\begin{array}{l}
2-22-2 \\
1-11-1
\end{array}\right)::\right)}, X_{(-211-211 \cdots)}\right\}, \\
& \lambda\left(D_{n}\right)=O\left(\frac{1}{n^{2}}\right), \quad\left|q_{n}\right|=O(n) \quad \text { for } \quad\left(\begin{array}{lll}
a_{1} \cdots & a_{n} \\
b_{1} \cdots & b_{n}
\end{array}\right) \in \mathscr{D}_{n} .
\end{aligned}
$$

Therefore

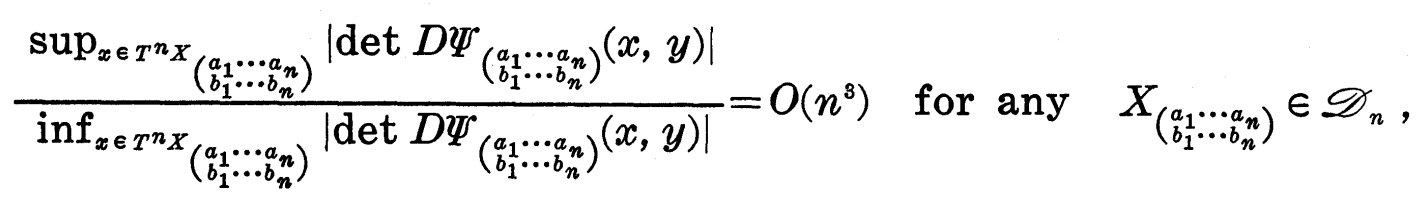

and if we put $\rho_{n}=\sup _{(x, y) \in D_{n}^{c}}\{|x|\}$, then $W_{n} \leqq \sum_{m=1}^{\infty} O\left(1 / m^{3}\right) \cdot\left(1 /\left(1-\rho_{n}\right)^{3}\right)$. Since $\operatorname{det} D T(x, y)=1 / x^{3}$, a direct calculation allows us to verify (C.8). Consequently, all of conditions of Theorem 3 are satisfied.

Finally, we consider a complex continued fraction transformation considered by S. Tanaka [17].

EXAMPLE 3. Let $X=\{z=x \alpha+y \bar{\alpha}:-1 / 2 \leqq x, y \leqq 1 / 2\}(\alpha=1+i)$ and define the transformation $T$ on $X$ by

$$
T z=\frac{1}{z}-\left[\frac{1}{z}\right]_{1},
$$

where $[z]_{1}$ denotes $[x+1 / 2] \alpha+[y+1 / 2] \bar{\alpha}$ for a complex number $z=x \alpha+y \bar{\alpha}$. Let $I=\{n \alpha+m \bar{\alpha}: m, n \in Z\} \backslash\{0\}$. The map $T$ induces a continued fraction expansion of $z \in X$,

$$
z=\frac{1 \mid}{\mid a_{1}}+\frac{1 \mid}{\mid a_{2}}+\cdots+\frac{1 \mid}{\mid a_{n}}+\cdots
$$

where each $a_{i}$ is contained in $I$. In his paper [17], he obtained the den- 
sity function of the invariant measure which is unbounded, the ergodicity, and some limiting values by his own method. His method cannot apply to general case. Here, applying our theory we obtain further results, i.e. exactness, Rohlin's formula, and a weak Bernoulli property.

Define $U_{j}(0 \leqq j \leqq 4)$ by

$$
\begin{aligned}
& U_{0}=X, \quad U_{1}=\left\{z \in X:\left|z+\frac{\alpha}{2}\right| \geqq \frac{1}{\sqrt{2}}\right\}, \quad U_{2}=-i \times U_{1}, \\
& U_{3}=-i \times U_{2} \quad U_{4}=-i \times U_{3} .
\end{aligned}
$$

From the above definitions it is easy to see that $T$ is a multidimensional map with a finite range structure, and Conditions (C.2) and (C.8) are satisfied. For (C.1), the detailed proof may be found in [17]. Define $q_{n} \in I(n \geqq-1)$ inductively by

$$
q_{-1}=0, \quad q_{0}=\alpha, \quad q_{n}=a_{n} q_{n-1}+q_{n-2} \quad(n \geqq 1) .
$$

Then a simple calculation gives the relation

$$
\left|\operatorname{det} D \Psi_{a_{1} \cdots a_{n}}(z)\right|=\frac{1}{\left|\boldsymbol{q}_{n}\right|^{4}\left|1+\left(q_{n-1} / q_{n}\right) z\right|^{4}}
$$

so that we can take Renyi's constant, $C=5^{4}$, and immediately we also verify (C.3) and (C.6) (see [17]). To examine (C.9), we show in Table 1, the admissibility of sequences $\left(a_{1} \cdots a_{n}\right)$ for which $X_{a_{1} \cdots a_{n}} \in \mathscr{D}_{n}$. This table shows that $k_{0}=3$ and $\mathscr{D}_{n}=4(4 n-3)$.

To estimate $\lambda\left(D_{n}\right)$, we remark that it is sufficient to estimate $\lambda\left(D_{n}^{\prime}\right)$, where

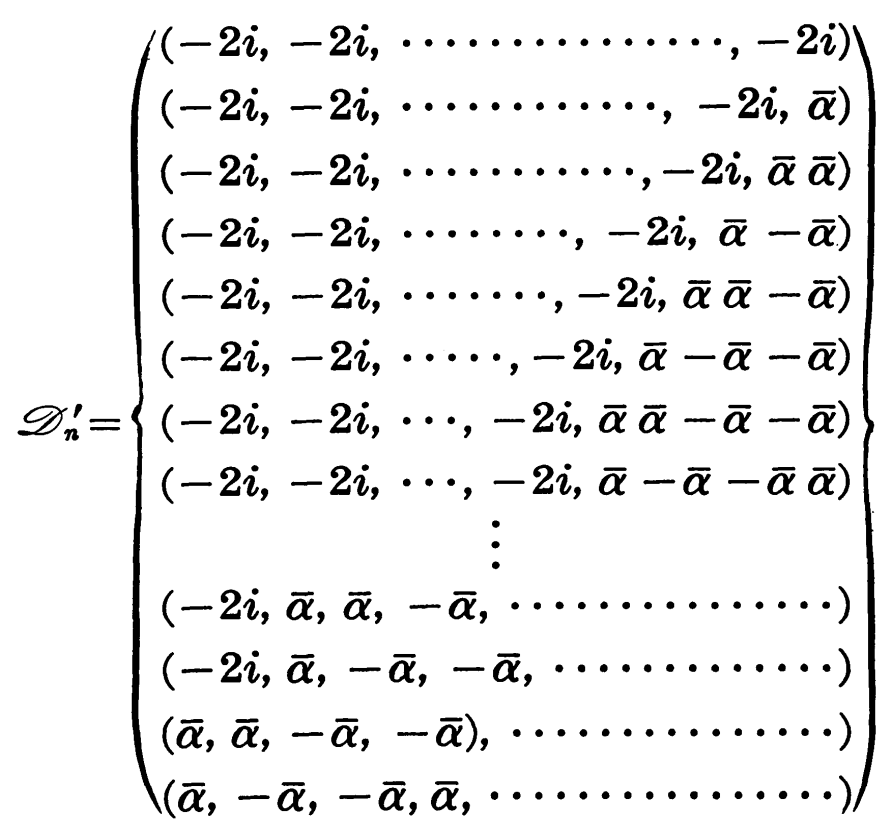


TABLE 1

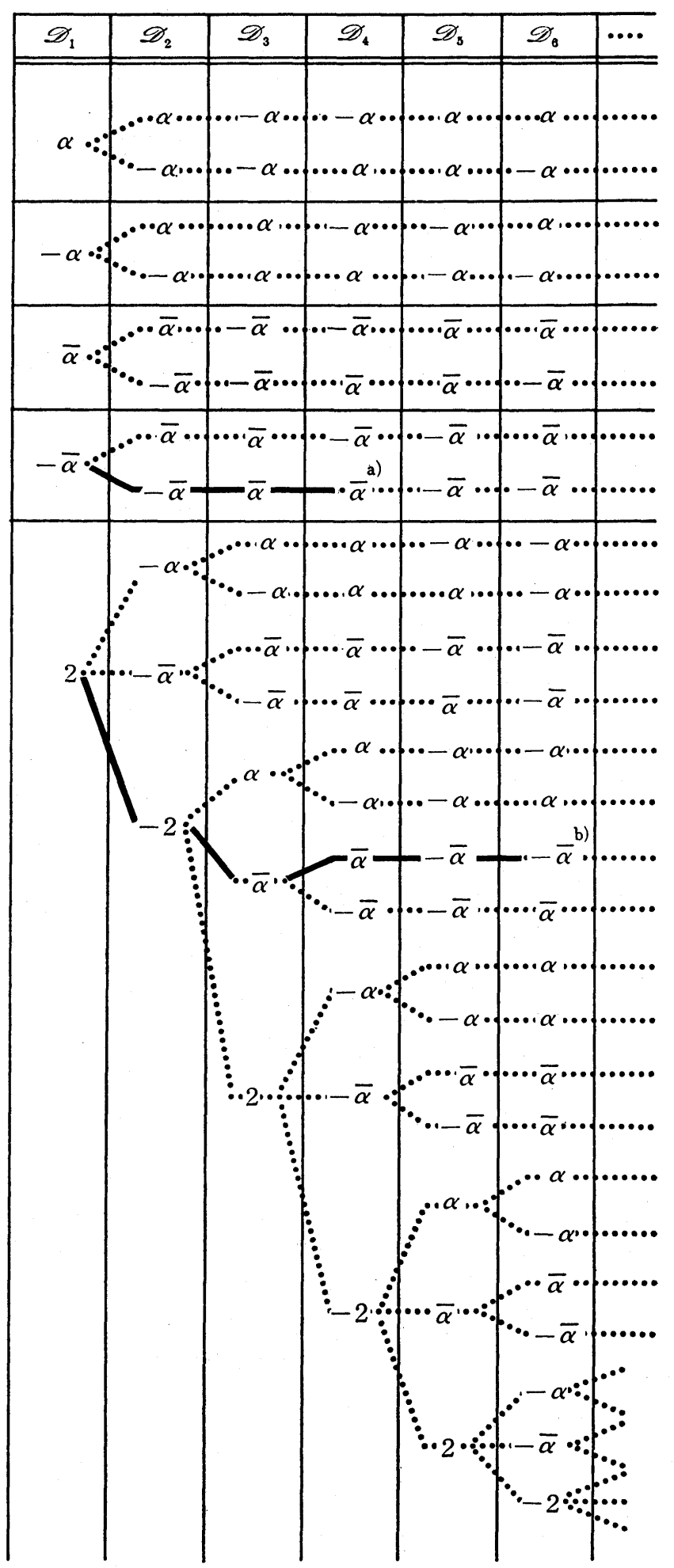

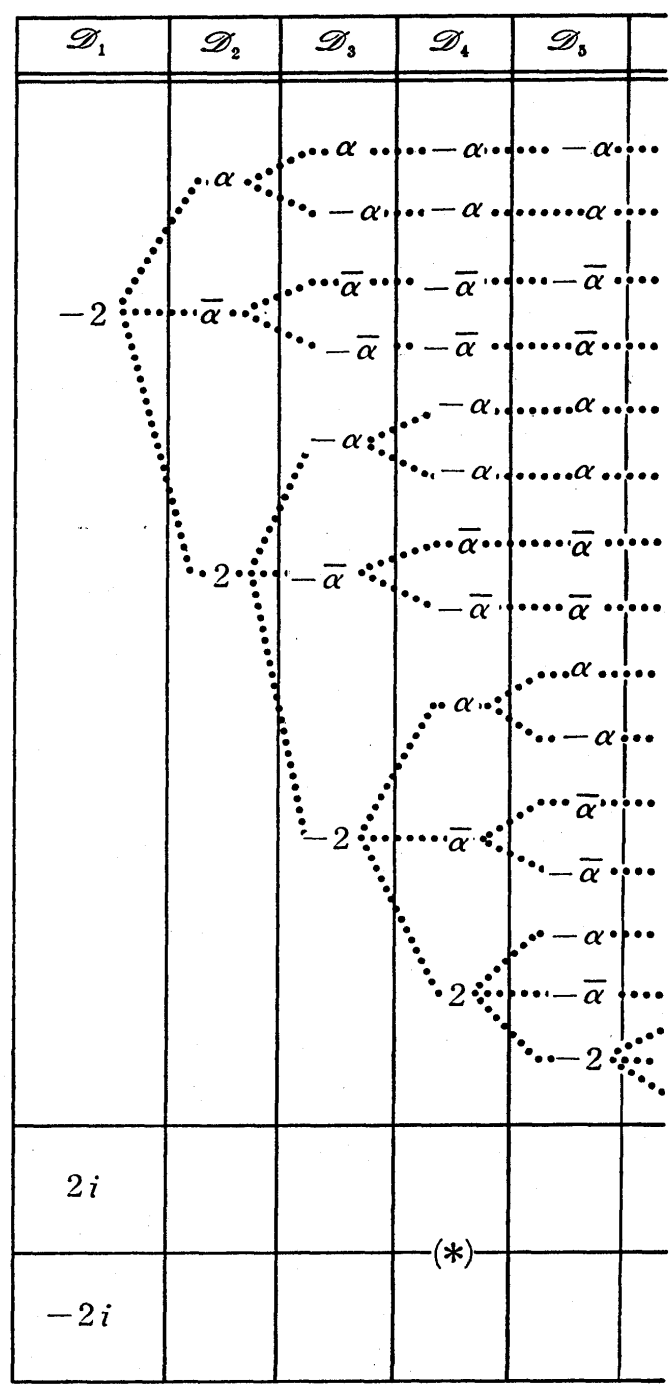

(1) In this table 1 , the sequence of symbols $\left.(-\bar{\alpha},-\bar{\alpha}, \bar{\alpha}, \bar{\alpha})^{a}\right)$

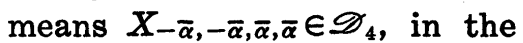
same way, the sequence $(2,-2$, $\bar{\alpha}, \bar{\alpha},-\bar{\alpha},-\bar{\alpha})^{b)}$ means

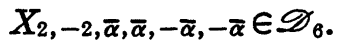

(2) ${ }^{(*)}$ The trees for $2 i$ and $-2 i$ are quite similar to trees for 2 and -2 , and hence are omitted. 
and

$$
D_{n}^{\prime}=\bigcup_{X_{a_{1} \cdots a_{n}} \in \mathscr{T}}^{\prime} X_{a_{1} \cdots a_{n}}
$$

Let $O(a, b, c)$ be a disc centered at $(a, b)$ with the radius $c$. Since

$$
D_{n}^{\prime} \subset O\left(\frac{1}{2 n}, \frac{2 n-1}{2 n}, \frac{\sqrt{2}}{2 n}\right) \cup O\left(\frac{2 n-1}{2 n}, \frac{1}{2 n}, \frac{\sqrt{2}}{2 n}\right) \text { (See Figure 3.), }
$$

we have $\lambda\left(D_{n}^{\prime}\right) \approx O\left(1 / n^{2}\right)$ and so $\lambda\left(D_{n}\right) \approx O\left(1 / n^{2}\right)$. Therefore (C.4)* is satisfied.

From the inequality

$$
\frac{\sup _{z \in T^{n} X_{a 1} \cdots a n}\left|\operatorname{det} D \Psi_{a_{1} \cdots a_{n}}(z)\right|}{\inf _{z \in T^{n} X_{a_{1} \cdots a_{n}}}\left|\operatorname{det} D \Psi_{a_{1} \cdots a_{n}}(z)\right|} \leqq \frac{\left(1+\left|q_{n-1} / q_{n}\right|\right)^{4}}{\left(1-\left|q_{n-1} / q_{n}\right|\right)^{4}},
$$

to verify (C.7), it is sufficient to estimate $\left|q_{n-1} / q_{n}\right|$. A direct calculation of $\left|q_{n}\right|$ seems to be very complicated, but the author has achieved to obtain
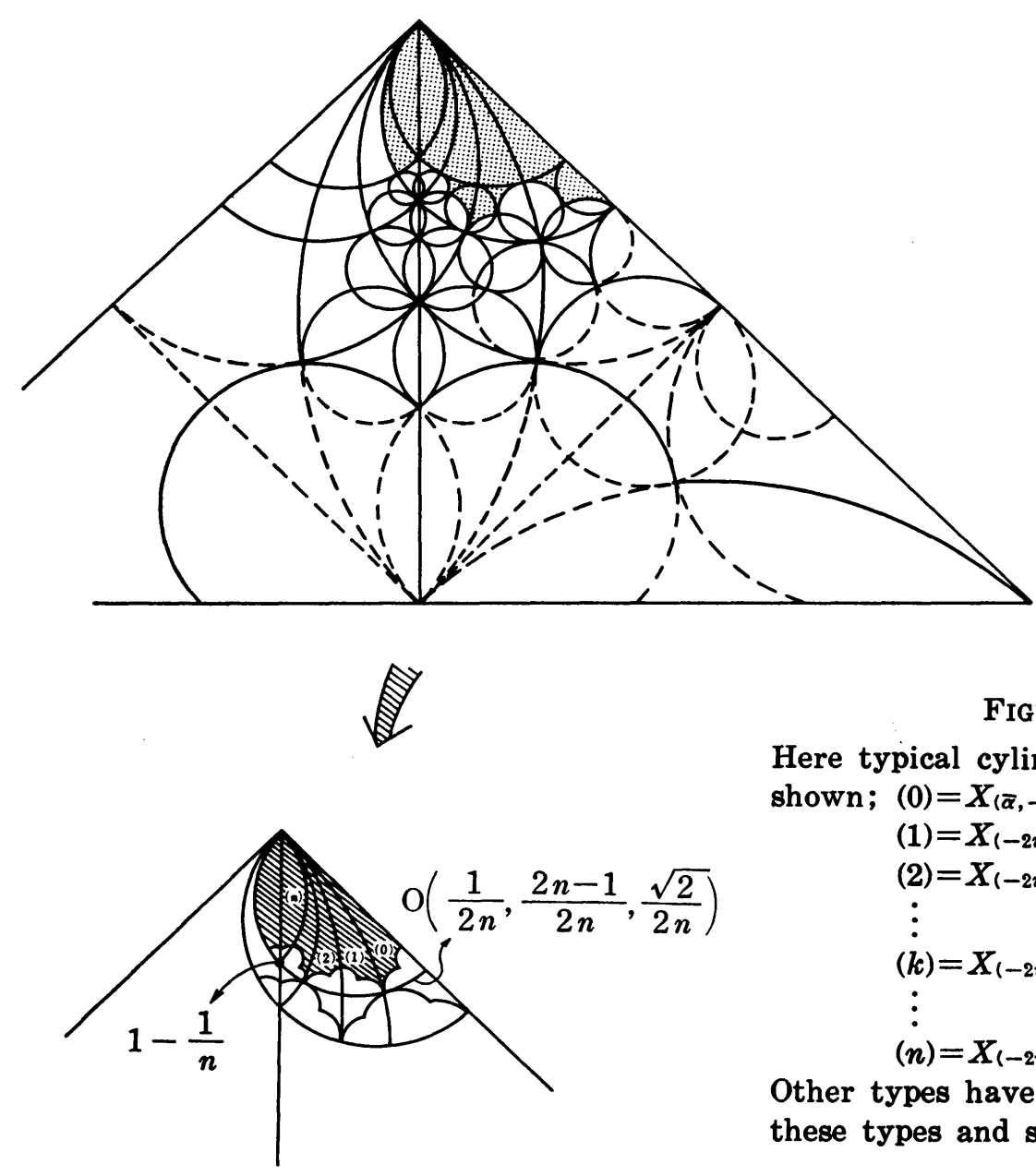

Figure 3

Here typical cylinders of $\mathscr{D}_{n}$ are shown; $(0)=X_{(\bar{\alpha},-\bar{\alpha},-\bar{\alpha} \cdots)}$

(1) $=X_{(-2 i, \bar{\alpha},-\bar{\alpha},-\bar{\alpha} \cdots)}$

(2) $=X_{(-2 i,-2 i, \bar{\alpha},-\bar{\alpha} \cdots)}$

:

$(k)=X(-2 i,-2 i \cdots-2 i, \bar{\alpha},-\bar{\alpha} \cdots \cdot)$

$(n)=X_{(-2 i,-2 i \cdots-2 i)}$

Other types have the same forms as these types and so are omitted. 
the following table:

TABLE 2

\begin{tabular}{c||l}
\hline \multicolumn{1}{c||}{ type } & \multicolumn{1}{c}{$\left|q_{n}\right|$} \\
\hline$(\alpha, \alpha,-\alpha,-\alpha, \cdots)$ & $\left|q_{2 k-1}\right|=2 k,\left|q_{2 k}\right|=\sqrt{4 k^{2}+4 k+2}$ \\
$(\bar{\alpha}, \bar{\alpha},-\bar{\alpha},-\bar{\alpha}, \cdots)$ & $\left|q_{2 k-1}\right|=2 \sqrt{k^{2}-2 k+2}, \quad\left|q_{2 k}\right|=\sqrt{2 k^{2}-2 k+10}$ \\
$(2 i, 2 i, \cdots)$ & \\
$(2,-2,2,-2, \cdots)$ & $\mid$ \\
$(-2 i,-2 i, \cdots)$ & $\left|q_{n}\right|=\sqrt{2}(n+1)$ \\
\hline
\end{tabular}

Since the type $(\cdots, 2 i, \alpha, \alpha,-\alpha,-\alpha, \cdots)$ is complicated, before we estimate $\left|q_{n}\right|$ we give a table of $q_{n}$ as follows:

TABLE 3

\begin{tabular}{|c|c|c|c|c|}
\hline type & $n=4 l$ & $n=4 l+1$ & $n=4 l+2$ & $n=4 l+3$ \\
\hline \multirow[t]{2}{*}{$2 i \cdots 2 i \alpha$} & $\begin{array}{l}(4 l+1) \\
\quad+i(-4 l+1)\end{array}$ & $4 l+i(4 l+2)$ & $\begin{array}{l}(-4 l-3) \\
+i(4 l+1)\end{array}$ & $\begin{array}{l}(-4 l-2) \\
\quad+i(-4 l-4)\end{array}$ \\
\hline & $(l \geqq 1)$ & $(l \geqq 1)$ & $(l \geqq 0)$ & $(l \geqq 0)$ \\
\hline $2 i \cdots 2 i \alpha \alpha$ & $\begin{array}{l}(-4 l+3) \\
\quad+i(-12 l+3)\end{array}$ & $12 l+i(-4 l+2)$ & $\begin{array}{l}(4 l-1) \\
\quad+i(12 l+3)\end{array}$ & $(-12 l-6)+i 4 l$ \\
\hline \multirow[t]{2}{*}{$2 i \cdots 2 i \alpha-\alpha$} & $\begin{array}{l}(-4 l-1) \\
\quad+i(4 l-1)\end{array}$ & $\begin{array}{l}(-4 l) \\
\quad+i(-4 l-2)\end{array}$ & $\begin{array}{l}(4 l+3) \\
\quad+i(-4 l-1)\end{array}$ & $\begin{array}{l}(4 l+2) \\
+i(4 l+4)\end{array}$ \\
\hline & $(l \geqq 1)$ & $(l \geqq 1)$ & $(l \geqq 1)$ & $(l \geqq 0)$ \\
\hline $2 i \cdots 2 i \alpha \alpha-\alpha$ & $\begin{array}{l}(12 l-9) \\
\quad+i(12 l-5)\end{array}$ & $\begin{array}{l}(-12 l+2) \\
\quad+i(12 l-6)\end{array}$ & $\begin{array}{l}(-12 l+3) \\
\quad+i(-12 l-1)\end{array}$ & $\begin{array}{l}(12 l+4) \\
\quad+i(-12 l)\end{array}$ \\
\hline \multirow[t]{2}{*}{$2 i \cdots 2 i \alpha-\alpha-\alpha$} & $\begin{array}{l}(-4 l+3) \\
\quad+i(-4 l-1)\end{array}$ & $\begin{array}{l}(4 l+2) \\
\quad+i(-4 l+2)\end{array}$ & $\stackrel{(4 l-1)}{+i(4 l+3)}$ & $(-4 l-4)+i 4 l$ \\
\hline & $(l \geqq 1)$ & $(l \geq 1)$ & $(l \geqq 1)$ & $(l \geqq 1)$ \\
\hline $2 i \cdots 2 i \alpha \alpha-\alpha-\alpha$ & $\begin{array}{l}(-20 l+15) \\
\quad+i(12 l-13)\end{array}$ & $\begin{array}{l}(-12 l+10) \\
\quad+i(-20 l+10)\end{array}$ & $\begin{array}{l}(20 l-5) \\
\quad+i(-12 l+7) \\
\end{array}$ & $(12 l-4)+i 20 l$ \\
\hline \multirow[t]{2}{*}{$2 i \cdots 2 i \alpha-\alpha-\alpha \alpha$} & $\begin{array}{l}(-4 l+3) \\
\quad+i(-4 l-1)\end{array}$ & $\begin{array}{l}(4 l+2) \\
\quad+i(-4 l+2)\end{array}$ & $\stackrel{(4 l-1)}{+i(4 l+3)}$ & $(-4 l-4)+i 4 l$ \\
\hline & $(l \geqq 2)$ & $(l \geqq 1)$ & $(l \geqq 1)$ & $(l \geqq 1)$ \\
\hline $2 i \cdots 2 i \alpha \alpha-\alpha-\alpha \alpha$ & $\begin{array}{l}(-20 l+19) \\
\quad+i(20 l-25)\end{array}$ & $\begin{array}{l}(-20 l+20) \\
\quad+i(-20 l+4)\end{array}$ & $\begin{array}{l}(20 l-9) \\
\quad+i-(20 l+15)\end{array}$ & $\begin{array}{l}(20 l-10) \\
\quad+i(20 l-4)\end{array}$ \\
\hline \multirow[t]{2}{*}{$2 i \cdots 2 i \alpha-\alpha-\alpha \alpha \alpha$} & $\begin{array}{l}(-4 l-1) \\
\quad+i(4 l-5)\end{array}$ & $\begin{array}{l}(-4 l+4) \\
\quad+i(-4 l-2)\end{array}$ & $\begin{array}{l}(4 l+3) \\
\quad+i(-4 l+3)\end{array}$ & $\begin{array}{r}(4 l-2) \\
+i(4 l+4)\end{array}$ \\
\hline & $(l \geqq 2)$ & ${ }^{(l \geqq 2)}$ & $:^{(l \geqq 1)}$ & $(l \geqq 1)$ \\
\hline
\end{tabular}


Therefore, we have the following:

$$
\begin{aligned}
& (2 i, 2 i, \cdots, 2 i, \underbrace{\alpha, \alpha, \cdots)}_{m} \quad \mid \begin{array}{ll}
m=2 k & \left|q_{4 l+j}\right|=\sqrt{\left\{32\left(4 m^{2}+1\right)\right\} l^{2}+O(l)} \\
m=2 k+1 & \left|q_{4 l+j}\right|=\sqrt{\left\{32(2 m+1)^{2}\right\} l^{2}+O(l)}
\end{array}
\end{aligned}
$$

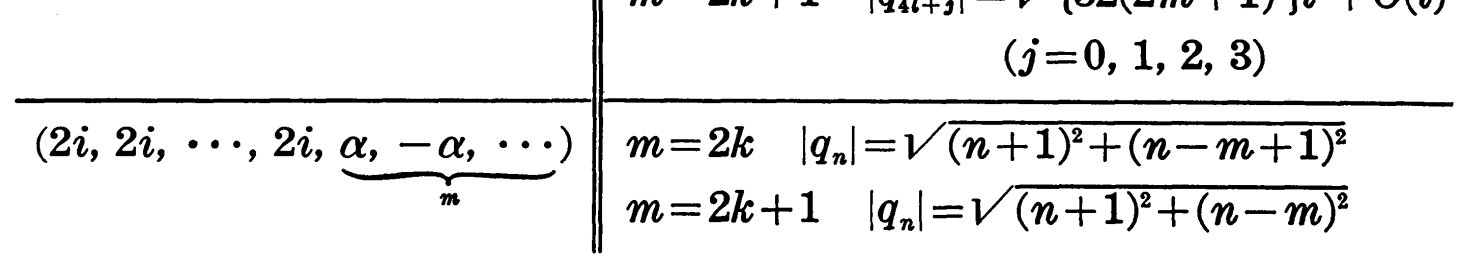

Other types are quite similar to one of the above types, and so are omitted. These allow us to verify (C.7).

Let $\rho_{n}=\sup _{\boldsymbol{z} \in D_{n}^{c}}\{|z|\}$. Then it is easy to see that

$$
W_{n} \leqq\left\{\sum_{m=0}^{\infty} \mathscr{D}_{m} O\left(\frac{1}{m^{4}}\right)\right\} \cdot \frac{1}{\left(1-\rho_{n}\right)^{4}}=\left\{\sum_{m=0}^{\infty} O\left(\frac{1}{m^{3}}\right)\right\} \cdot \frac{1}{\left(1-\rho_{n}\right)^{4}} \cdot
$$

This implies (C.5).

\section{References}

[1] P. Billingsley, Ergodic Theory and Information, J. wiley New York, 1965.

[2] R. Bowen, Bernoulli maps of the interval, Israel J. Math., 28 (1977), 161-168.

[3] K. L. Chung, A note on the ergodic theorem of information theory, Ann. Math. Statistics, 32 (1961), 612-614.

[4] SH. ITO and M. YURI, Number theoretical transformations with finite range structure and their ergodic properties, to appear.

[5] Sн. Iто, Diophantine approximation in inhomogeneous linear class and its metrical theory, to appear.

[6] F. LEDRAPPIER, Some properties of absolutely continuous invariant measures on an interval, Ergodic Theory \& Dynamical Systems, 1 (1981), 77-93.

[7] F. LEDRAPPIER, Principe variational et systems dynamiques simboliques, Z. Wahrsch. Verw. Gebiete, 30 (1974), 185-202.

[8] F. LeDrappier, Sur la condition de Bernoulli faible et ses applications, Lecture Notes in Math., 532 (1976), Springer 154-159.

[9] H. NAKADA, On the Kuzmin's theorem for complex continued fractions, KEIO Engineering Report, 29 (1976), 93-108.

[10] D. S. ORnstein and B. Weiss, Geodesic flows are Bernoullian, Israel J. Math., 14 (1973), 184-198.

[11] Y.B. PESIN, Description of the $\Pi$-partition of a diffeomorphism with invariant smooth measure, Mat. Zametki, vol. 21, no. 6, (1977), 29-44.

[12] M. RATNER, Anosov flows are also Bernoullian, Israel J. Math., 17 (1974), 380-391.

[13] V.I. RoHLIN, Exact endomorphism of a Lebesgue spaces, Amer. Math. Soc. Transl, Ser 2, 39 (1964), 1-36.

[14] F. SCHWEIGER, Some remarks on ergodicity and invariant measures, Michigan Math. J., 22 (1975), 181-187.

[15] Y. G. SinaI, Markov partitions and $C$-diffeomorphisms, Functional Anal. Appl., 2 (1968), 64-89. 
[16] Y. G. SinaI, Gibbs measure in ergodic theory, Russian Math. Surveys, vol. 27, no. 4, (1972), 21-69.

[17] S. TANAKA, A complex continued fraction transformation and its ergodic properties, Tokyo J. Math., 8 (1985), 191-214.

[18] M.S. WATERMaN, Some ergodic properties of multi-dimensional $f$-expansion, Z. Wahrsch. Verw. Gebiete, 16 (1977), 77-103.

Present Address:

DEPARTMENT OF MATHEMATICS

TSuda College

TSUDA-MACHI, KODAIRA, TOKYO 187 